\title{
Design of Orthogonal Filtered Multitone Modulation Systems and Comparison among Efficient Realizations
}

\author{
Nicola Moret and Andrea M. Tonello (EURASIP Member) \\ Dipartimento di Ingegneria Elettrica Gestionale e Meccanica (DIEGM), Università degli Studi di Udine, \\ Via delle Scienze 208, 33100, Udine, Italy \\ Correspondence should be addressed to Andrea M. Tonello, tonello@uniud.it
}

Received 8 July 2009; Revised 19 October 2009; Accepted 31 December 2009

Academic Editor: Faouzi Bader

Copyright ( $\odot 2010$ N. Moret and A. M. Tonello. This is an open access article distributed under the Creative Commons Attribution License, which permits unrestricted use, distribution, and reproduction in any medium, provided the original work is properly cited.

\begin{abstract}
We address the efficient realization of a filtered multitone (FMT) modulation system and its orthogonal design. FMT modulation can be viewed as a Discrete Fourier Transform (DFT) modulated filter bank (FB). It generalizes the popular orthogonal frequency division multiplexing (OFDM) scheme by deploying frequency confined subchannel pulses. We compare three realizations that have been described by Cvetković and Vetterli (1998), and Weiss and Stewart (2000), and Tonello (2006). A detailed derivation of them is performed in the time-domain via the exploitation of different FB polyphase decompositions. We then consider the design of an orthogonal FMT system and we exploit the third realization which allows simplifying the orthogonal FB design and obtaining a block diagonal system matrix with independent subblocks. A numerical method is then presented to obtain an orthogonal FB with well frequency confined subchannel pulses for arbitrarily large number of subchannels. Several examples of pulses with minimal length are reported and their performance is evaluated in typical multipath fading channels. Finally, we compare the orthogonal FMT system with a cyclically prefixed OFDM system in the IEEE 802.11 wireless LAN channel. In this scenario, FMT with minimal length pulses and single tap subchannel equalization outperforms the OFDM system in achievable rate.
\end{abstract}

\section{Introduction}

Multicarrier (MC) systems deploy a transmission technique where a high rate information signal is transmitted through a wide band channel by simultaneous modulation of a set of parallel signals at low rate. The parallel signals are obtained by the serial-to-parallel $(\mathrm{S} / \mathrm{P})$ conversion of the input information signal. The idea dates back 50 years ago [1] and it has originated by the goal of simplifying the equalization task in highly frequency selective channels that introduce severe intersymbol interference (ISI). This is made possible because the wide band channel is divided in a number of narrow band subchannels that exhibit a nearly flat frequency response. If the number of subchannels is sufficiently large, and the intercarrier interference (ICI) is negligible, a single tap equalizer per subchannel will be sufficient for data detection.

The MC transmitter is implemented using a synthesis filer bank (FB) while the MC receiver uses an analysis $\mathrm{FB}$.
When the modulation is accomplished with an exponential function, we obtain the so-called exponentially modulated FB. It is also referred to as Discrete Fourier Transform FB (DFT-FB) since, as it is also shown in this paper, the efficient realization exploits a DFT.

MC modulation systems have been adopted in several wireless communication standards as the WLAN IEEE 802.11 and the WMAN IEEE 802.16 standards. MC modulation is also considered for application in $4 \mathrm{G}$ systems. The most popular MC architecture is orthogonal frequency division multiplexing (OFDM) [2]. It can be viewed as an exponentially modulated FB with a prototype pulse that has a rectangular impulse response. Another scheme is Filtered Multitone (FMT) modulation. It differs from OFDM since it uses confined frequency response pulses. FMT has been originally proposed for application in broadband wireline channels [3], and subsequently it has been investigated for application in wireless channels [4] and in power line communications [5]. 
The main research problems related to FMT are the efficient digital implementation, the design of the prototype pulse, the development of equalization schemes, the synchronization problem, and in general the performance analysis and comparison with other schemes. This paper focuses on the first two aspects.

We consider three efficient realizations that have been described: one by Tonello in [6], one by Cvetković and Vetterli in the context of signal coding [7], (and more recently by Siclet et al. in [8] and by McGee in [9]), and another one by Weiss and Stewart in [10]. They are all based on the deployment of a DFT and a polyphase FB network. In this paper, we aim at describing the differences and similarities. Instead of deriving the realizations in the $Z$ domain, as it is conventionally done, we operate in the time domain which allows explicitly detailing the implementation steps. We also compute the complexity of the architectures in terms of number of operations per second. The three methods require similar memory space and achieve identical reductions in complexity but differ in the elegance of their representation.

The time-frequency selectivity of the wireless channel may introduce ICI and ISI that can be minimized with the design of time-frequency confined pulses. Then, if the ICI is negligible, we can cope with the residual interference either via simplified subchannel equalization [3] or optimal and iterative multichannel equalization $[4,11]$. The design of well-localized pulses in analog MC systems has been treated by several authors [12-17]. A method for the design of quasiorthogonal DFT modulated FB has been proposed in [18]. Recently, simple pulse design criteria for nonorthogonal FMT have been reported in [19].

In [20] we have studied the performance limits of FMT modulation, and we have given design guidelines such that frequency and time diversity gains are attainable with optimal multichannel equalization. However, if complexity is an issue, linear single channel equalizers will be desirable. Their performance in doubly dispersive fading channels has been studied in [21] assuming a rectangular, a sinc, a rootraised-cosine (rrc), and a Gaussian pulse.

The FB design described in [12-19] does not provide orthogonality using finite length pulses, that is, the FB does not grant perfect reconstruction even in the presence of an ideal channel. This condition eases the pulse design problem. The goal of achieving orthogonality is desirable but it makes the FB design more challenging. The construction of orthogonal DFT filter banks has been discussed in [7, 8]. Examples of pulses with practical relevance have been reported in [8] where, however, it is recognized that the design becomes significantly complex as the number of subchannels increases. Therefore, it has been proposed to perform polynomial fitting of the parameters to decrease the number of variables which however yields a quasiorthogonal FB. In [22] we have presented preliminary results about the perfect orthogonal FB design. In this paper, we bring new insights to this problem. We show that the polyphase decomposition of the signals used in the realization [6] allows deriving the orthogonal FB equations and writing them in a number of uncoupled subsets. Each subset needs a small number of parameters, which simplifies the search of optimal pulses. The search of optimal pulses is carried out with the objective of maximizing the inband to total pulse energy, or minimizing the mean square error between the pulse and a target frequency response. A numerical method is presented to obtain well frequency confined subchannel pulses for an arbitrary large number of subchannels. Some examples of pulses with minimal length are then reported.

Finally, the performance in terms of average signal-tointerference power ratio of the orthogonal FMT system in typical multi-path fading channels is reported and compared to that obtained with a conventional truncated root-raisedcosine pulse. Then, we report a comparison in terms of achievable rate between FMT and cyclically prefixed OFDM using the IEEE 802.11 WLAN channel model in [23]. It is found that even deploying minimal length pulses and with single tap equalization, in the considered scenario, the achievable rate of FMT is higher than that of OFDM.

This paper is organized as follows. In Section 2, we report the notation used in this paper. In Section 3, we describe the FMT system model, while in Sections 4, 5 and 6 we derive the three efficient realizations. The differences/similarities and the complexity analysis is reported in Section 7. The construction of an orthogonal FMT system is discussed in Section 8. Several design examples are reported in Section 9. The performance in fading channels is shown in Section 10. Finally, we report the conclusions.

\section{Notation}

The notation related to the operators, constants, and signals used in this paper is summarized in Table 1. A discrete time signal is denoted either with $x$ or $x(N n)$. It is a function $x: Z(N) \rightarrow \mathbb{C}$ where $Z(N)$ is the set of integer numbers multiple of $N$ with $N$ belonging to the set $\mathbb{N}$ of natural numbers, that is, $Z(N)=\{-\infty, \ldots,-N, 0, N, 2 N, \ldots,+\infty\}$, and $\mathbb{C}$ is the set of complex numbers. The notation $x(N n)$ explicitly shows the definition domain of the signal.

2.1. Operators. To derive the FMT realizations presented in this paper, it is convenient to use the notation of operators, in particular, the translation, sampling, and interpolation. They are defined as follows.

(1) Operator $\tau^{a}$ : translation of signal $x: Z(P) \rightarrow \mathbb{C}$ by $a \in \mathbb{Z}$

$$
\tau^{a}[x]=x(P(n+a)) .
$$

(2) Operator $\mathcal{C}_{N}$ : sampling of signal $x: Z(P) \rightarrow \mathbb{C}$ by $N \in \mathbb{N}$

$$
\mathcal{C}_{N}[x]=x(N P n)
$$

(3) Operator $\ell_{N}$ : interpolation of signal $y: Z(K) \rightarrow \mathbb{C}$ by $N \in \mathbb{Z}$, with $K=P N$, and $P \in \mathbb{N}$

$$
\ell_{N}[y]= \begin{cases}y(P n) & \text { if } n \in Z(N), \\ 0 & \text { otherwise. }\end{cases}
$$


TABLE 1: Operator notation and useful signals and constants.

\begin{tabular}{|c|c|}
\hline \multicolumn{2}{|c|}{ Operator Notation } \\
\hline Convolution & {$[x * h](n)$} \\
\hline Translation & $\begin{array}{c}\tau^{a}[x](P n)=x(P(n+a)) \\
(x: Z(P) \rightarrow \mathbb{C})\end{array}$ \\
\hline Sampling & $\begin{array}{c}\mathcal{C}_{N}[x](N P n)=x(N P n) \\
(x: Z(P) \rightarrow \mathbb{C})\end{array}$ \\
\hline Interpolation & $\begin{array}{r}\boldsymbol{l}_{N}[y](P n)= \begin{cases}y(P n) & \text { if } n \in Z(N) \\
0 & \text { otherwise }\end{cases} \\
(y: Z(K) \rightarrow \mathbb{C} \text { and } K=N P)\end{array}$ \\
\hline \multicolumn{2}{|c|}{ Signals and constants } \\
\hline$M$ & number of subchannels \\
\hline$N$ & sampling-interpolation factor \\
\hline$M_{1}$ & l.c.m. $(M, N)$ (least common multiple) \\
\hline$M_{0}$ & $M_{1} / N$ \\
\hline$N_{0}$ & $M_{1} / M$ \\
\hline$L_{f}$ & prototype filter length \\
\hline$L_{M}$ & $L_{f} / N$ \\
\hline$L_{N}$ & $L_{f} / M$ \\
\hline$W_{M}$ & $e^{-j(2 \pi / M)}$ \\
\hline$a^{(k)}(N n)$ & data input at the $k$ th subchannel \\
\hline$b^{(k)}(N n)$ & output at the $k$ th subchannel \\
\hline$g(n)$ & synthesis bank prototype filter \\
\hline$h(n)$ & analysis bank prototype filter \\
\hline$\tilde{a}^{(k)}(N n)$ & $a^{(k)}(N n) W_{M}^{k N n}$ \\
\hline$\tilde{b}^{(k)}(N n)$ & $b^{(k)}(N n) W_{M}^{-k N n}$ \\
\hline$g^{(k)}(n)$ & $g(n) W_{M}^{-k n}$ \\
\hline$h^{(k)}(n)$ & $h(n) W_{M}^{-k n}$ \\
\hline $\operatorname{div}[A, B]$ & floor $(A / B)$ \\
\hline $\bmod [A, B]$ & $A-\operatorname{div}[A, B] B$ \\
\hline $\begin{array}{l}y_{a}=\sum_{b=0}^{N_{0}-1} x_{a+M b} \\
a \in\{0, \ldots, M-1\}\end{array}$ & $\begin{array}{l}M \text {-periodic repetition of } x_{i} \\
\quad i \in\left\{0, \ldots, N_{0} M-1\right\}\end{array}$ \\
\hline $\begin{array}{l}x_{a}=y_{\bmod [a, M]} \\
a \in\left\{0, \ldots, N_{0} M-1\right\}\end{array}$ & $\begin{array}{c}N_{0} \text {-cyclic extension of } y_{i} \\
i \in\{0, \ldots, M-1\}\end{array}$ \\
\hline
\end{tabular}

We often write signals with the specification of the definition domain, for example, $\tau^{a}[x](P n)$ instead of $\tau^{a}[x]$, as in Table 1. The properties of the operators that are exploited in this paper are listed below.

(1) Translation properties:

$$
\begin{aligned}
\tau^{a}[x+y] & =\tau^{a}[x]+\tau^{a}[y] \quad \text { Additive property, } \\
\tau^{a}[x y] & =\tau^{a}[x] \tau^{a}[y] \quad \text { Multiplicative property, } \\
\tau^{a}\left[\tau^{b}[x]\right] & =\tau^{b}\left[\tau^{a}[x]\right] \\
& =\tau^{a+b}[x] \quad \text { Commutative property. }
\end{aligned}
$$

(2) Sampling properties:

$$
\begin{aligned}
\mathcal{C}_{M}[x+y] & =\mathcal{C}_{M}[x]+\mathcal{C}_{M}[y] \quad \text { Additive property, } \\
\mathcal{C}_{M}[x y] & =\mathcal{C}_{M}[x] \mathcal{C}_{M}[y] \quad \text { Multiplicative property, } \\
\mathcal{C}_{M}\left[\mathcal{C}_{N}[x]\right] & =\mathcal{C}_{N}\left[\mathcal{C}_{M}[x]\right] \\
& =\mathcal{C}_{M N}[x] \quad \text { Commutative property, } \\
\mathcal{C}_{N}\left[\tau^{N a}[x]\right] & =\tau^{a}\left[\mathcal{C}_{N}[x]\right]
\end{aligned}
$$$$
\text { Sampling-translation property. }
$$

(3) Interpolation properties:

$$
\begin{aligned}
\ell_{M}[x+y] & =\ell_{M}[x]+\ell_{M}[y] \quad \text { Additive property, } \\
\ell_{M}[x y] & =\ell_{M}[x] \ell_{M}[y] \quad \text { Multiplicative property, } \\
\ell_{M}\left[\ell_{N}[x]\right] & =\ell_{N}\left[\ell_{M}[x]\right] \\
& =\ell_{M N}[x] \quad \text { Commutative property, } \\
\ell_{N}\left[\tau^{a}[x]\right]= & \tau^{N a}\left[\ell_{N}[x]\right] \\
& \text { Interpolation-translation property. }
\end{aligned}
$$

(4) Convolution properties:

$$
\begin{aligned}
\tau^{a}[x * y] & =x * \tau^{a}[y] \\
& =\tau^{a}[x] * y
\end{aligned}
$$

Convolution-translation property,

$$
\begin{aligned}
\ell_{N}[x] * \ell_{N}[h] & =\ell_{N}[x * h] \quad \text { Noble identity } 1, \\
\mathcal{C}_{N}\left[x * \ell_{N}[h]\right] & =\mathcal{C}_{N}[x] * h \quad \text { Noble identity 2, } \\
x *\left(h w^{(k)}\right) & =\left(\left(x w^{(-k)}\right) * h\right) w^{(k)}
\end{aligned}
$$

Convolution-modulation property,

$$
\text { where in }(18) w^{(k)}(n)=W_{M}^{k n} \text { and } W_{M}=e^{-j(2 \pi / M)} \text {. }
$$

2.2. Polyphase Decomposition. The M-order polyphase decomposition of a signal $x: Z(P) \rightarrow \mathbb{C}$ generates $M$ low-rate signals $x_{i}: Z(M P) \rightarrow \mathbb{C}$ with $i \in\{0,1, \ldots, M-1\}$ that are referred to as polyphase components. They are defined as

$$
\begin{aligned}
x_{i}(M P n) & =x(P(M n+i)) \\
& =\mathcal{C}_{M}\left[\tau^{i}[x]\right] \quad \text { A-type polyphase decomposition. }
\end{aligned}
$$

The polyphase components are obtained with a serial-toparallel $(\mathrm{S} / \mathrm{P})$ conversion. We can recover the original signal $x$ from the polyphase components as follows:

$$
x(P n)=\sum_{i=0}^{M-1} \ell_{M}\left[x_{i}\right](P n-P i)=\sum_{i=0}^{M-1} \tau^{-i}\left[\ell_{M}\left[x_{i}\right]\right](P n) .
$$




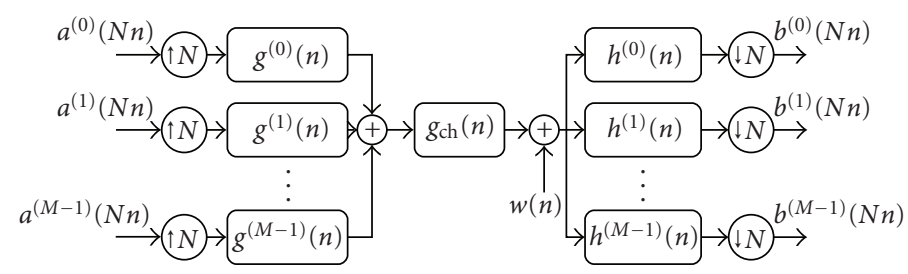

FIGURE 1: Modified FMT scheme.

Relation (20) corresponds to a parallel-to-serial $(\mathrm{P} / \mathrm{S})$ conversion. If we change $i$ into $-i$, the polyphase decomposition will be referred to as B-type decomposition.

\section{FMT Scheme}

We consider an FMT scheme as depicted in Figure 1 where the discrete-time transmitted signal at the output of the synthesis $\mathrm{FB}, x: Z(1) \rightarrow \mathbb{C}$, is obtained by the modulation of $M$ data streams at low rate $a^{(k)}: Z(N) \rightarrow \mathbb{C}$, with $k \in\{0,1, \ldots, M-1\}$, that belong to the $Q A M$ signal set. Using the operator notation, as summarized in Table 1, the transmitted signal can be written as

$$
x(n)=\sum_{k=0}^{M-1} \sum_{l \in \mathbb{Z}} a^{(k)}(N l) g^{(k)}(n-N l)=\sum_{k=0}^{M-1}\left[\ell_{N}\left[a^{(k)}\right] * g^{(k)}\right](n),
$$

where $M$ is the number of subchannels of the transmitter, and $N$ is the sampling-interpolation factor. According to (21), the signals $a^{(k)}(N l)$ are upsampled by a factor $N$ and are filtered by the modulated pulses $g^{(k)}(n)=g(n) W_{M}^{-k n}$, with $g(n)$ being the prototype filter of the synthesis bank and $W_{M}^{k n}=e^{-j(2 \pi / M) k n}$. Then, the subchannel signals are summed and sent over the transmission media.

After propagation through the transmission media, the received signal $y(n)$ is processed by the analysis $\mathrm{FB}$ whose outputs are

$$
b^{(k)}(N n)=\sum_{m \in \mathbb{Z}} y(m) h^{(k)}(N n-m)=\mathcal{C}_{N}\left[y * h^{(k)}\right](N n) .
$$

We refer to the direct implementation of (21)-(22) as the inefficient realization since it requires a bank of highrate filters. Fortunately, the FMT scheme can be efficiently realized via three DFT based architectures that we describe in the following.

\section{Realization A: $M_{1}$-Order Polyphase Decomposition of the Signals}

4.1. Synthesis Bank in Method A. A first efficient realization of the synthesis bank [6] will be derived if we perform a polyphase decomposition of order $M_{1}=M_{0} N=N_{0} M=$ l.c.m. $[M, N]$ of the signal $x$ in (21). The ith polyphase component $x_{i}: Z\left(M_{1}\right) \rightarrow \mathbb{C}$ with $i \in\left\{0, \ldots, M_{1}-1\right\}$ can be written as

$$
\begin{aligned}
x_{i}\left(M_{1} n\right) & =\sum_{k=0}^{M-1} \sum_{l \in \mathbb{Z}} a^{(k)}(N l) g\left(M_{1} n+i-N l\right) W_{M}^{-k\left(M_{1} n+i-N l\right)} \\
& =\sum_{l \in \mathbb{Z}} \sum_{k=0}^{M-1} a^{(k)}(N l) W_{M}^{k N l} g_{i}\left(M_{1} n-N l\right) W_{M}^{-k i}
\end{aligned}
$$

where $g_{i}: \mathbb{Z}(N) \rightarrow \mathbb{C}$ is the $i$ th $N$-order polyphase decomposition of the pulse $g$. If we define

$$
\begin{gathered}
\tilde{a}^{(k)}(N l)=a^{(k)}(N l) W_{M}^{k N l}, \\
\tilde{A}^{(i)}(N l)=\sum_{k=0}^{M-1} \tilde{a}^{(k)}(N l) W_{M}^{-k i},
\end{gathered}
$$

where $\tilde{a}^{(k)}(N l)$ is the signal obtained by modulating the data symbols by $W_{M}^{k N l}$, and $\widetilde{A}^{(i)}: \mathbb{Z}(N) \rightarrow \mathbb{C}$ for $i \in\left\{0, \ldots, M_{1}-\right.$ $1\}$ is the Inverse Discrete Fourier Transform (IDFT) of the signal $\tilde{a}^{(k)}$ for $k \in\{0, \ldots, M-1\}$, we will obtain

$$
\begin{aligned}
x_{i}\left(M_{1} n\right) & =\sum_{l \in \mathbb{Z}} \widetilde{A}^{(i)}(N l) g_{i}\left(M_{0} N n-N l\right) \\
& =\mathcal{C}_{M_{0}}\left[\tilde{A}^{(i)} * g_{i}\right]\left(M_{1} n\right) .
\end{aligned}
$$

Now, in order to better understand the structure of the polyphase filters, we use the operator notation which helps us to greatly simplify the derivation. First, we redefine the index $i$ as

$$
i=\alpha+N \beta=p+M m
$$

with

$$
\begin{array}{ll}
\alpha=\bmod [i, N], & \beta=\operatorname{div}[i, N], \\
p=\bmod [i, M], & m=\operatorname{div}[i, M]
\end{array}
$$

and $\alpha \in\{0, \ldots, N-1\}, \beta \in\left\{0, \ldots, M_{0}-1\right\}, p \in\{0, \ldots, M-$ $1\}, m \in\left\{0, \ldots, N_{0}-1\right\}$.

Then, we have that $\widetilde{A}^{(i)}=\widetilde{A}^{(p+m M)}=\widetilde{A}^{(p)}=\widetilde{A}^{(\bmod [i, M])}$ and

$$
\begin{aligned}
g_{i} & =g_{\alpha+N \beta} \\
& =\mathcal{C}_{N}\left[\tau^{\alpha+N \beta}[g]\right] \quad(\text { using (19)) } \\
& =\tau^{\beta}\left[\mathcal{C}_{N}\left[\tau^{\alpha}[g]\right]\right] \quad(\text { using (5) and (10)) } \\
& =\tau^{\operatorname{div}[i, N]}\left[g_{\bmod [i, N]}\right]
\end{aligned}
$$

(using (19) and substituting $\alpha$ and $\beta$ ). 


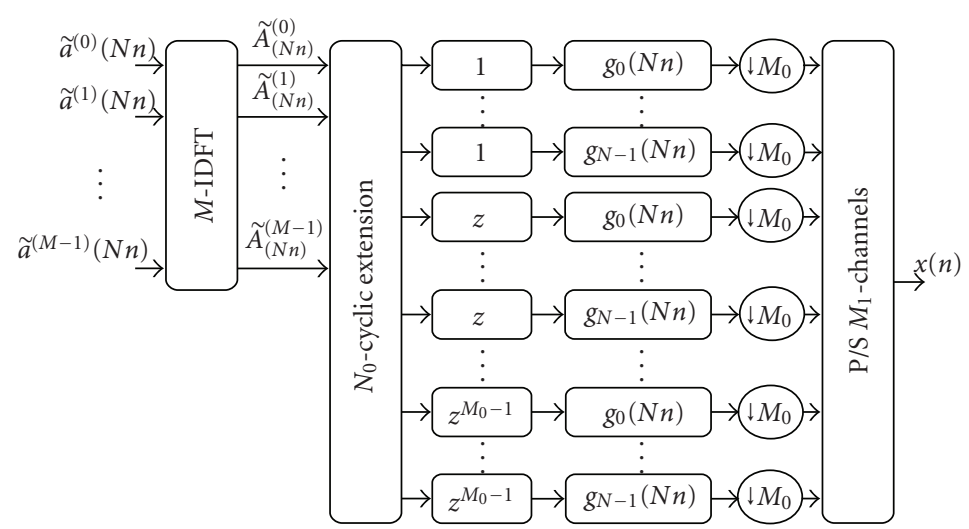

FIgURE 2: Synthesis bank (method A).

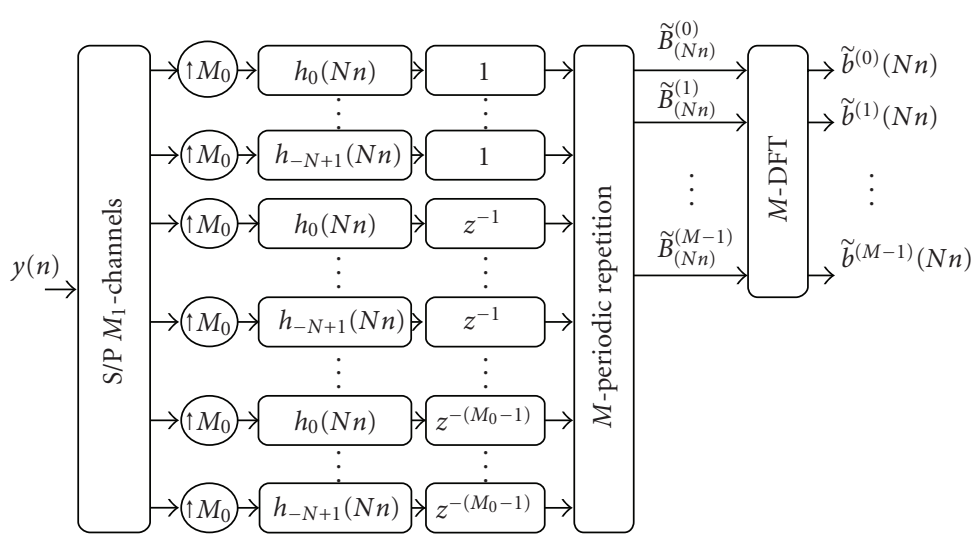

FIgURe 3: Analysis bank (method A).

Finally, the $i$ th polyphase component of the transmitted signal $x$ can be written as

$$
x_{i}\left(M_{1} n\right)=\mathcal{C}_{M_{0}}\left[\tilde{A}^{(\bmod [i, M])} * \tau^{\operatorname{div}[i, N]}\left[g_{\bmod [i, N]}\right]\right]\left(M_{1} n\right) .
$$

Therefore, as shown in Figure 2, the synthesis FB realization comprises the following operations. The blocks of data $\tilde{a}^{(k)}$ are processed by an $M$-point IDFT. Each output block is cyclically extended to the block $\tilde{A}^{(\bmod [i, M])}$ of size $M_{1}$. The signals $\widetilde{A}^{(i)}$ are filtered, after a delay, with the $N$ order polyphase components of the prototype pulse. Finally, the filter outputs are sampled by a factor $M_{0}$ and parallel-toserial converted.

4.2. Analysis Bank in Method A. According to [6], the efficient realization of the analysis FB is obtained with an $M_{1}$ order polyphase decomposition of the signal $y(n) W_{M}^{k n}$ at the receiver. We can rearrange the analysis bank equation (22) as

$$
\begin{aligned}
b^{(k)}(N n) & =\sum_{i \in \mathbb{Z}} y(i) h(N n-i) W_{M}^{-k(N n-i)} \\
& =\left(\sum_{i \in \mathbb{Z}} y(i) W_{M}^{k i} h(N n-i)\right) W_{M}^{-k N n} .
\end{aligned}
$$

Now, if we define

$$
\tilde{b}^{(k)}(N n)=\sum_{i \in \mathbb{Z}} y(i) W_{M}^{k i} h(N n-i)
$$

and we perform a polyphase decomposition of order $M_{1}=$ l.c.m. $(M, N)=M_{0} N=N_{0} M$ on the signal $y(i) W_{M}^{k i}$, we will obtain

$$
\begin{aligned}
\tilde{b}^{(k)}(N n) & =\sum_{l=0}^{M_{1}-1} \sum_{i \in \mathbb{Z}} y\left(M_{1} i+l\right) W_{M}^{k\left(M_{1} i+l\right)} h\left(N n-M_{1} i-l\right) \\
& =\sum_{l=0}^{M_{1}-1} \sum_{i \in \mathbb{Z}} y_{l}\left(M_{1} i\right) h_{-l}\left(N n-M_{1} i\right) W_{M}^{k l} \\
& =\sum_{l=0}^{M_{1}-1}\left[l_{M_{0}}\left[y_{l}\right] * h_{-l}\right](N n) W_{M}^{k l}
\end{aligned}
$$

If we redefine $l=p+M m=\alpha+N \beta$ with $m \in$ $\left\{0, \ldots, N_{0}-1\right\}, p \in\{0, \ldots, M-1\}, \alpha \in\{0, \ldots, N-$ $1\}$, and $\beta \in\left\{0, \ldots, M_{0}-1\right\}$ as in (27)-(28), we will 
obtain

$$
\begin{aligned}
h_{-l}= & h_{-\alpha-N \beta} \\
= & \mathcal{C}_{N}\left[\tau^{-\alpha-N \beta}[h]\right] \quad(\text { using }(19)) \\
= & \tau^{-\beta}\left[\mathcal{C}_{N}\left[\tau^{-\alpha}[h]\right]\right] \quad(\text { using }(5) \text { and }(10)) \\
= & \tau^{-\operatorname{div}[l, N]}\left[h_{-\bmod [l, N]}\right] \\
& (\text { using (19) and substituting } \alpha \text { and } \beta) .
\end{aligned}
$$

Substituting (39) in (35), we obtain the final expression as follows

$$
\begin{aligned}
& \tilde{b}^{(k)}(N n)= \\
& \sum_{p=0}^{M-1} W_{M}^{k p}\left(\sum _ { m = 0 } ^ { N _ { 0 } - 1 } \left[\ell_{M_{0}}\left[y_{p+M m}\right]\right.\right. \\
& \\
&\left.\left.\quad * \tau^{-\operatorname{div}[p+M m, N]}\left[h_{-\bmod [p+M m, N]}\right]\right](N n)\right) .
\end{aligned}
$$

Therefore, as shown in Figure 3, the analysis FB realization comprises the following operations. The received signal is serial-to-parallel converted with a converter of size $M_{1}$. The output signals are upsampled by a factor $M_{0}$, filtered with the $N$-order polyphase components of the prototype pulse. Then, after a delay, we compute the periodic repetition with period $M$ of the block of coefficients of size $M_{1}$. Finally, the $M$-point DFT is performed.

\section{Realization B: $M_{1}$-order Polyphase Decomposition of the Pulses}

5.1. Synthesis Bank in Method B. In a second method [7, 8], the efficient realization of the synthesis FB is obtained by performing an $M_{1}$-order polyphase decomposition of the filter $g^{(k)}$, with $M_{1}=$ l.c.m $(M, N)$. In Appendix A, a detailed derivation in the time-domain is reported and it shows that the polyphase components $x_{\alpha}: \mathbb{Z}(N) \rightarrow \mathbb{C}$, with $\alpha \in$ $\{0,1, \ldots, N-1\}$, of the transmitted signal $x$ can be written as (see Appendix A)

$$
x_{\alpha}(N n)=\sum_{\beta=0}^{M_{0}-1}\left[A^{(\bmod [\alpha+N \beta, M])} * \tau^{-\beta}\left[\ell_{M_{0}}\left[g_{\alpha+N \beta}\right]\right]\right](N n) .
$$

In (41), $A^{(l)}: Z(N) \rightarrow \mathbb{C}$, with $l \in\{0,1, \ldots, M-1\}$, is the $M$-point IDFT of $a^{(k)}, k \in\{0, \ldots, M-1\}$, that is,

$$
A^{(l)}(N n)=\sum_{k=0}^{M-1} a^{(k)}(N n) W_{M}^{-k l}
$$

and $g_{i}: \mathbb{Z}\left(M_{1}\right) \rightarrow \mathbb{C}$ are the $M_{1}$-order polyphase components of the prototype filter $g$ that are defined as

$$
g_{i}\left(M_{1} n\right)=\mathcal{C}_{M_{1}}\left[\tau^{i}[g]\right]\left(M_{1} n\right) \quad i \in\left\{0,1, \ldots, M_{1}-1\right\}
$$

Therefore, as shown in Figure 4, the synthesis FB realization comprises the following operations. The blocks $a^{(k)}$ of data are processed with an $M$-IDFT. The output block is cyclically extended to a block of size $M_{1}$. The subchannel signals are filtered, after a delay, with the $M_{1}$-order polyphase components of the prototype pulse interpolated by $M_{0}$. The output blocks of size $M_{1}$ are periodically repeated with period $N$ and parallel-to-serial converted by a converter of size $N$.

5.2. Analysis Bank in Method B. In this second method [7, 8], the efficient realization of the analysis FB is obtained by exploiting the $M_{1}$-order polyphase decomposition of the pulses $h^{(k)}$ with $k \in\{0,1, \ldots, M-1\}$. The FB outputs can be written as (see Appendix A)

$$
\begin{array}{r}
b^{(k)}(N n)= \\
\sum_{p=0}^{M-1}\left(W _ { M } ^ { k p } \sum _ { m = 0 } ^ { N _ { 0 } - 1 } \left[\tau^{\mathrm{div}[p+M m, N]} y_{\bmod [p+M m, N]}\right.\right. \\
\left.\left.* \ell_{M_{0}}\left[h_{-p-M m}\right]\right](N n)\right),
\end{array}
$$

where $y_{i}: \mathbb{Z}(N) \rightarrow \mathbb{C}$ are the $N$-order polyphase components of the received signal $y$ that are defined as

$$
y_{i}(N n)=\mathcal{C}_{N}\left[\tau^{i}[y]\right](N n) \quad i \in\{0,1, \ldots, N-1\} .
$$

The $M_{1}$-order polyphase components of the prototype filter $h$ are defined as

$$
h_{-l}\left(M_{1} n\right)=\mathcal{C}_{M_{1}}\left[\tau^{-l}[h]\right]\left(M_{1} n\right) \quad l \in\left\{0,1, \ldots, M_{1}-1\right\} .
$$

Therefore, as shown in Figure 5, this realization comprises the following operations. The received signal is serialto-parallel converted by a size $N$ converter. The outputs of the $\mathrm{S} / \mathrm{P}$ converter are cyclically extended $M_{0}$ times. Then, the signals are filtered with the $M_{1}$-order polyphase components of the prototype pulse after appropriate delays. Finally, a periodic repetition with period $M$ on the output blocks is computed, and an $M$-DFT is performed.

\section{Realization C: $L_{f}$-Order Polyphase Decomposition of the Pulses}

6.1. Synthesis Bank in Method C. The third method of realizing the synthesis FB is described in [10]. It starts from the assumption that the prototype pulse $g$ has length $L_{f}=L_{M} N=L_{N} M$, that is, without loss of generality, a multiple of both $M$ and $N$. Then, if we exploit the $L_{f}$-order polyphase decomposition of the filters $g^{(k)}$, each having a single coefficient, the $\alpha$ th $N$-order polyphase component of the signal $x$ can be written as

$$
x_{\alpha}(N n)=\sum_{\beta=0}^{L_{M}-1} \tau^{-\beta}\left[A^{(\bmod [\alpha+N \beta, M])} \times g(\alpha+N \beta)\right](N n) .
$$




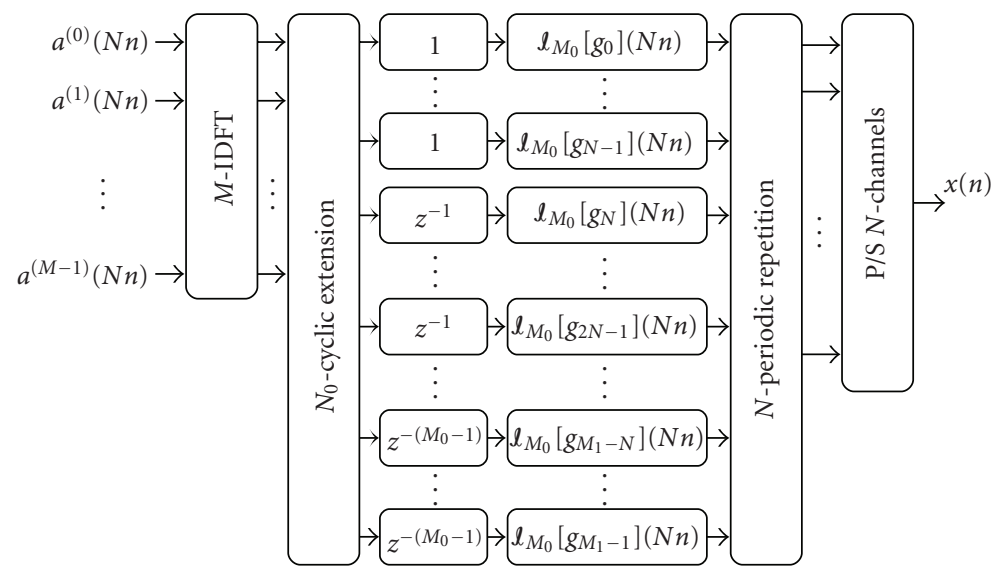

FIgURE 4: Synthesis bank (method B).

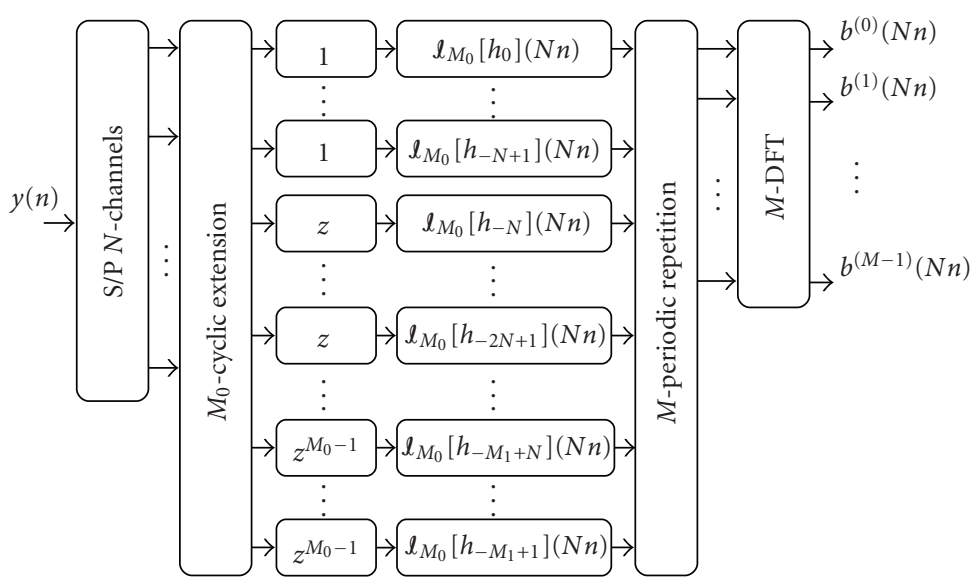

Figure 5: Analysis bank (method B).

In (47), $A^{(l)}: Z(N) \rightarrow \mathbb{C}$, with $l \in\{0,1, \ldots, M-1\}$, is the $M$-point IDFT of $a^{(k)}, k \in\{0, \ldots, M-1\}$, (see (45)) and $g(i)$ are the prototype pulse coefficients that correspond to the $L_{f}$-order polyphase components. The proof is given in Appendix B.

Therefore, as shown in Figure 6, the realization comprises the following operations. The data signals $a^{(k)}$ are processed with an $M$-IDFT. The output blocks are cyclically extended, to form a block of size $L_{N} M$. Then, the outputs after a proper delay are multiplied by the polyphase coefficients of the prototype filter. Each output block is periodically repeated with period $N$, and parallel-to-serial converted with a converter of size $N$.

6.2. Analysis Bank in Method C. We assume, according to [10], without loss of generality the pulse $h(n)$ to be anticausal and defined for $n \in\left\{-L_{f}+1, \ldots, 0\right\}$. The efficient realization of the analysis FB is obtained exploiting the $L_{f}$ order polyphase decomposition of the filter $h^{(k)}$ with $k \in$ $\{0,1, \ldots, M-1\}$. In Appendix B, we show that the FB outputs are obtained as follows:

$$
\begin{aligned}
& b^{(k)}(N n)= \\
& \sum_{p=0}^{M-1} W_{M}^{k p}\left(\sum _ { m = 0 } ^ { L _ { N } - 1 } \tau ^ { \operatorname { d i v } [ p + M m , N ] } \left[y_{\bmod [p+M m, N]}\right.\right. \\
& \times h(-p-M m)](N n)),
\end{aligned}
$$

where $y_{i}: \mathbb{Z}(N) \rightarrow \mathbb{C}$ are the $N$-order polyphase components of the received signal $y$ that are defined as

$$
y_{i}(N n)=\mathcal{C}_{N}\left[\tau^{i}[y]\right](N n) \quad i \in\{0,1, \ldots, N-1\}
$$

and $h(i)$ are the prototype pulse coefficients that correspond to the $L_{f}$-order polyphase components.

Therefore, as shown in Figure 7, the received signal is serial-to-parallel converted with a converter of size $N$. The outputs of the S/P converter are cyclically extended $M_{0}$ times. Then, the signals are delayed and multiplied with 


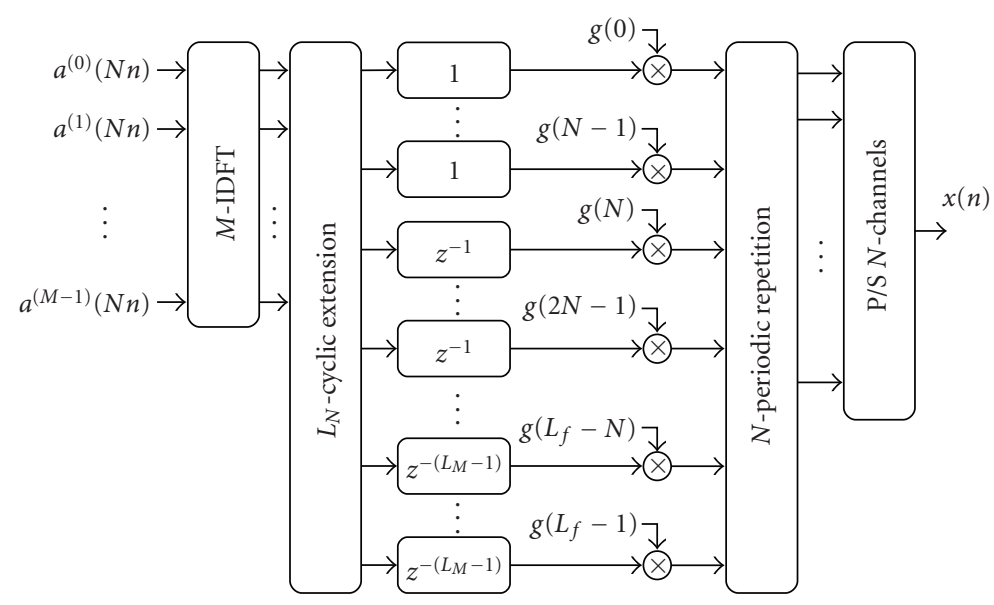

FIgure 6: Synthesis bank (method C).

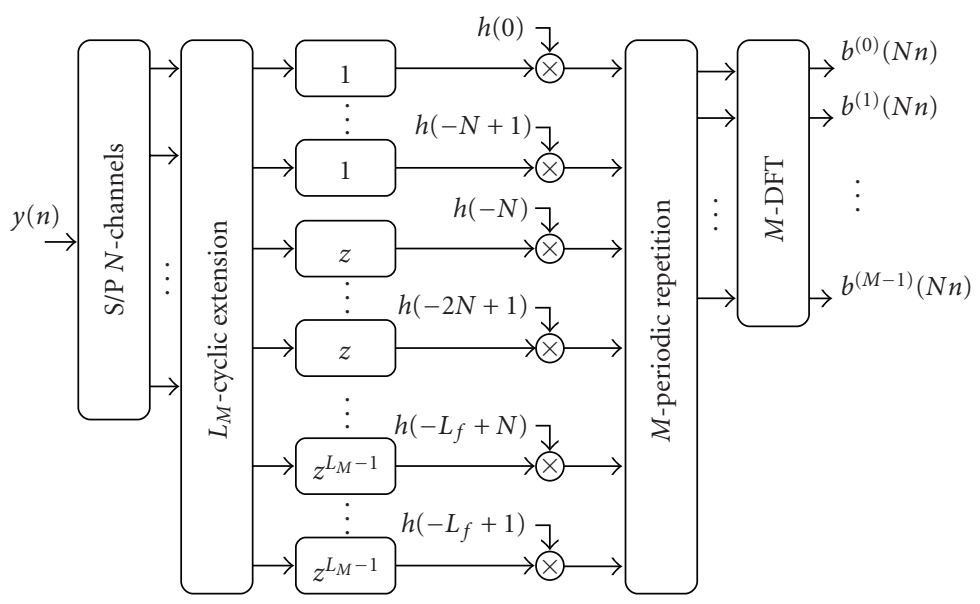

Figure 7: Analysis bank (method C).

the coefficients of the prototype filter. The resulting block is periodically repeated with period $M$, and finally, an $M$-DFT is applied.

\section{Comparison among FMT Realizations}

All three realizations deploy an $M$-point DFT, and essentially differ in the MIMO polyphase FB network which has size $M_{1} \times M_{1}$ in the first and second realizations, while it has size $L_{f} \times L_{f}$ in the third realization. Furthermore, the polyphase components of the pulses for methods $A, B$, and $C$ have different length that is respectively equal to $L_{f} / N, L_{f} / M_{1}$, and 1. When $L_{f}=M_{1}$, the implementations $B$ and $C$ are identical.

The complexity of the three structures in terms of number of complex operations (additions and multiplications) per unit sampling time is identical and it has order equal to $\left(\alpha M \log _{2} M+2 L_{f}\right) / N$ for both the synthesis and the analysis bank which can be proved following the detailed calculation for realization A in [5]. The factor $\alpha$ depends on the FFT algorithm [24]. As an example, assuming $M=64, N=80$, $\alpha=1.2$, and a pulse with length $L_{f} / N=\{1,2,3\}$, the complexity for realizations $\mathrm{A}, \mathrm{B}$, and $\mathrm{C}$ respectively equals $\{7.8,9.8,11.8\}$ oper./samp. for both the transmitter and the receiver.

The three schemes require the same memory usage that we define in terms of memory units per output sample (MUPS), where a memory unit is the space required to store one coefficient. Without taking into account the memory requirements of the DFT stages (identical for all three realizations), the synthesis and the analysis polyphase $\mathrm{FB}$ respectively require $\left(M L_{M}+N\right) / N$ and $\left(L_{f}+M\right) / M$ MUPS. As an example, assuming again $M=64, N=80$, and a pulse with length $L_{f} / N=\{1,2,3\}$, the MUPS for realizations $\mathrm{A}, \mathrm{B}$, and $\mathrm{C}$ are respectively equal to $\{1.8,2.6,3.4\}$ for the synthesis bank and to $\{2.3,3.5,4.8\}$ for the analysis bank. It should however to be noted that as discussed in [25], depending on the DSP architecture and the specific processing procedures, the memory requirements may slightly change. 
Another difference is that when we derive the perfect reconstruction (orthogonality) conditions in matrix form from the efficient realization, we obtain a different factorization of the system matrix that can be exploited in the design and search of optimal orthogonal pulses. This is discussed in the next section.

\section{Perfect Reconstruction and Orthogonality}

To derive the perfect reconstruction conditions for the FB, we can exploit the realization A of Figures 2 and 3. Perfect reconstruction will be achieved if the $M$-IDFT output coefficients at the transmitter $\widetilde{A}^{(k)}$ are identical (despite a delay) to the input block $\widetilde{B}^{(k)}$ of coefficients to the $M$-DFT at the receiver. The signal of the ath subchannel at the input of the DFT receiver is given by

$$
\widetilde{B}^{(a)}(N n)=\sum_{b=0}^{N_{0}-1}\left[\ell_{M_{0}}\left[x_{a+M b}\right] * h_{-a-M b}\right](N n) .
$$

The analysis subchannel pulse has been obtained by the the $N$-order polyphase decomposition of the prototype filter $h$, that is, $h_{l}=\mathcal{C}_{N}\left[\tau^{-l}[h]\right]$ with $l \in\left\{0,1, \ldots, M_{1}-1\right\}$. We now perform a further $M_{0}$-order polyphase decomposition of the subchannel pulse and we obtain that it equals the $M_{1}$-order polyphase component of $h$. This is shown in what follows:

$$
\begin{aligned}
\mathcal{C}_{M_{0}}\left[\tau^{\beta}\left[h_{-a-M b}\right]\right] & =\mathcal{C}_{M_{0}}\left[\tau^{\beta}\left[\mathcal{C}_{N}\left[\tau^{-a-M b}[h]\right]\right]\right. \\
& =\mathcal{C}_{M_{0}}\left[\mathcal{C}_{N}\left[\tau^{N \beta}\left[\tau^{-a-M b}[h]\right]\right]\right] \\
& =\mathcal{C}_{M_{1}}\left[\tau^{N \beta-a-M b}[h]\right]=h_{N \beta-a-M b}^{\prime} .
\end{aligned}
$$

Now, (50) can be rewritten as

$$
\begin{aligned}
\widetilde{B}^{(a)}(N n) & =\sum_{b=0}^{N_{0}-1}\left[\ell_{M_{0}}\left[x_{a+M b}\right] * \sum_{\beta=0}^{M_{0}-1} \tau^{-\beta}\left[\ell_{M_{0}}\left[h_{N \beta-a-M b}^{\prime}\right]\right](N n)\right. \\
& =\sum_{\beta=0}^{M_{0}-1} \tau^{-\beta}\left[\ell_{M_{0}}\left[\sum_{b=0}^{N_{0}-1} x_{a+M b} * h_{N \beta-a-M b}^{\prime}\right](N n)\right. \\
& =\sum_{\beta=0}^{M_{0}-1} \tau^{-\beta}\left[\ell_{M_{0}}\left[\widetilde{B}_{\beta}^{(a)}\right]\right](N n),
\end{aligned}
$$

where we have defined

$$
\begin{aligned}
\widetilde{B}_{\beta}^{(a)}\left(M_{1} n\right) & =\sum_{b=0}^{N_{0}-1}\left[x_{a+M b} * h_{N \beta-a-M b}^{\prime}\right]\left(M_{1} n\right) \\
& =\sum_{b=0}^{N_{0}-1}\left[\mathcal{C}_{M_{0}}\left[\tilde{A}^{(a+M b)} * g_{a+M b}\right] * h_{N \beta-a-M b}^{\prime}\right]\left(M_{1} n\right) .
\end{aligned}
$$

Similarly to what has been done for the pulses $h_{l}$, we can perform an $M_{0}$-order polyphase decomposition of the pulses $g_{l}$, and obtain that it equals the $M_{1}$-order polyphase decomposition of the prototype pulse $g$, that is,

$$
\mathcal{C}_{M_{0}}\left[\tau^{-\alpha}\left[g_{a+M b}\right]\right]=\mathcal{C}_{M_{1}}\left[\tau^{a+M b-N \alpha}[g]\right]=g_{a+M b-N \alpha}^{\prime} .
$$

It follows that (53) can be written as

$$
\begin{aligned}
\widetilde{B}_{\beta}^{(a)}\left(M_{1} n\right)=\sum_{b=0}^{N_{0}-1}\left[\mathcal{C}_{M_{0}}\left[\widetilde{A}^{(a)} * \sum_{\alpha=0}^{M_{0}-1} \tau^{\alpha}\left[\ell_{M_{0}}\left[g_{a+M b-N \alpha}^{\prime}\right]\right]\right]\right. \\
\left.* h_{N \beta-a-M b}^{\prime}\right]\left(M_{1} n\right) \\
=\sum_{\alpha=0}^{M_{0}-1}\left[\mathcal{C}_{M_{0}}\left[\tau^{\alpha}\left[\tilde{A}^{(a)}\right]\right] * \sum_{b=0}^{N_{0}-1} g_{a+M b-N \alpha}^{\prime}\right. \\
\left.* h_{N \beta-a-M b}^{\prime}\right]\left(M_{1} n\right) .
\end{aligned}
$$

Finally, if we define with $\widetilde{A}_{\alpha}^{(a)}=\mathcal{C}_{M_{0}}\left[\tau^{\alpha}\left[\tilde{A}^{(a)}\right]\right]$ the $M_{0}$ order polyphase component of $\widetilde{A}^{(a)}$, we can write

$$
\widetilde{B}_{\beta}^{(a)}\left(M_{1} n\right)=\sum_{\alpha=0}^{M_{0}-1}\left[\tilde{A}_{\alpha}^{(a)} * \sum_{b=0}^{N_{0}-1} g_{a+M b-N \alpha}^{\prime} * h_{N \beta-a-M b}^{\prime}\right]\left(M_{1} n\right),
$$

where the signal $\tilde{A}_{\alpha}^{(a)}: Z\left(M_{1}\right) \rightarrow \mathbb{C}$ and $\widetilde{B}_{\beta}^{(a)}: Z\left(M_{1}\right) \rightarrow \mathbb{C}$ with $\alpha, \beta \in\left\{0,1, \ldots, M_{0}-1\right\}$ are the $M_{0}$ order polyphase components of $\widetilde{A}^{(a)}$ and $\widetilde{B}^{(a)}$ :

$$
\tilde{A}_{\alpha}^{(a)}\left(M_{1} n\right)=\mathcal{C}_{M_{0}}\left[\tau^{\alpha} \tilde{A}^{(a)}\right]\left(M_{1} n\right) \quad \alpha \in\left\{0,1, \ldots, M_{0}-1\right\},
$$

$$
\widetilde{B}_{\beta}^{(a)}\left(M_{1} n\right)=\mathcal{C}_{M_{0}}\left[\tau^{\beta} \widetilde{B}^{(a)}\right]\left(M_{1} n\right) \quad \beta \in\left\{0,1, \ldots, M_{0}-1\right\},
$$

and $g_{i}^{\prime}: \mathbb{Z}\left(M_{1}\right) \rightarrow \mathbb{C}, h_{i}^{\prime}: \mathbb{Z}\left(M_{1}\right) \rightarrow \mathbb{C}$ with $i \in$ $\left\{0,1, \ldots, M_{1}-1\right\}$ are the $M_{0}$ order polyphase components of $g_{i}$ and $h_{i}$. They are equal to the $M_{1}$-order polyphase components of the prototype pulse $g$ and $h$, that is,

$$
\begin{aligned}
g_{i}^{\prime}\left(M_{1} n\right)= & \mathcal{C}_{M_{1}}\left[\tau^{i}[g]\right]\left(M_{1} n\right) \quad i \in\left\{0,1, \ldots, M_{1}-1\right\}, \\
& h_{i}^{\prime}\left(M_{1} n\right)=\mathcal{C}_{M_{1}}\left[\tau^{i}[h]\right]\left(M_{1} n\right) .
\end{aligned}
$$

Therefore, from (57) the perfect reconstruction condition becomes

$$
\sum_{b=0}^{N_{0}-1}\left[g_{M b+a-N \alpha}^{\prime} * h_{N \beta-a-M b}^{\prime}\right]\left(M_{1} n\right)=\delta\left(M_{1} n\right) \delta(\alpha-\beta)
$$

with $\delta(k)$ being the Kronecker delta. Applying the Ztransform to $(60)$, the relation becomes

$$
\sum_{b=0}^{N_{0}-1} G_{M b+a-N \alpha}^{\prime}(z) H_{N \beta-a-M b}^{\prime}(z)=\delta(\alpha-\beta) .
$$


Thus, if we define the following $M_{0} \times N_{0}$ matrix:

$$
\mathbf{G}_{a}(z)=\left[\begin{array}{ccc}
G_{a}^{\prime}(z) & \cdots & G_{\left(N_{0}-1\right) M+a}^{\prime}(z) \\
G_{a-N}^{\prime}(z) & \cdots & G_{\left(N_{0}-1\right) M+a-N}^{\prime}(z) \\
\vdots & \vdots & \vdots \\
G_{a-\left(M_{0}-1\right) N}^{\prime}(z) & \cdots & G_{\left(N_{0}-1\right) M+a-\left(M_{0}-1\right) N}^{\prime}(z)
\end{array}\right]^{T}
$$

and we assume a matched analysis $\mathrm{FB}$, that is, $\mathbf{H}_{-a}(z)=$ $\left(\mathbf{G}_{a}^{T}\left(1 / z^{*}\right)\right)^{*}$, the perfect reconstruction conditions will become the orthogonality conditions such that they can be written in matrix form as

$$
\mathbf{H}_{-a}(z) \mathbf{G}_{a}(z)=\mathbf{I}_{M_{0}} \quad a \in\{0,1, \ldots, M-1\} .
$$

It is interesting to note that the FB is orthogonal when every submatrix (63) is orthogonal. Further, when $M$ and $N$ are not prime among them, that is, l.c.m. $(M, N) \neq 1$, each submatrix contains pulse coefficients that are distinct from those in another submatrix. Consequently, the orthogonality conditions are imposed on distinct subsets of pulse coefficients.

We note that for $N=M$, the submatrices are squared therefore the only possible solution is to choose the prototype filter with length $M$ (submatrices are polynomial so their inverse is polynomial if and only if each submatrix component is a monomial). Therefore, a plausible solution is the rectangular pulse, which yields the OFDM scheme. If $N>M$, the submatrices become rectangular enhancing the degrees of freedom in the choice of the shape and the length of the prototype filter.

\section{Orthogonal FMT System Design}

Orthogonal matrices can be constructed via the parametrization of the pulse coefficients with angles as proposed in [8], that is, expressing the pulse coefficients with trigonometric functions of the angles. For every choice of the angles, the FB is orthogonal. Then, the search of optimal pulses can be done defining an objective function (metric). The metric can be defined either as the maximization of the in-band energy to the total pulse energy, or as the minimization of the mean squared error between the pulse spectrum and a target frequency response. The metrics are the following.

(1) METRIC 1: maximum in-band to total energy

$$
\widehat{\boldsymbol{\theta}}=\underset{\boldsymbol{\theta}}{\arg \max } \frac{\int_{-1 / 2 M}^{1 / 2 M}|G(f, \boldsymbol{\theta})|^{2} d f}{\int_{-\infty}^{\infty}|G(f, \boldsymbol{\theta})|^{2} d f},
$$

where $G(f, \boldsymbol{\theta})$ is the frequency response of the pulse obtained for a certain choice of the angles stored in the vector $\boldsymbol{\theta}$.

(2) METRIC 2: least squares

$$
\widehat{\boldsymbol{\theta}}=\underset{\boldsymbol{\theta}}{\arg \min } \int_{-\infty}^{\infty}|G(f, \boldsymbol{\theta})-H(f)|^{2} d f,
$$

where $H(f)$ is the target frequency response.
Since the efficient realization requires an $M$-point DFT, we can impose $M=2^{n}$ with $n$ integer in order to allow for an efficient fast Fourier transform-based implementation. Furthermore, since $N=2^{n} N_{0} / M_{0}$ must be integer, $M_{0}$ must also be an integer power of two. Now, we can choose $N_{0}=$ $M_{0}+1$ such that we minimize the amount of redundancy, that is, minimize the ratio $N / M=N_{0} / M_{0}$. Furthermore, we can choose $M$ and $N$ not to be relatively prime which simplifies the orthogonal FB design. If it is further desired to have nonpolynomial submatrices, we can choose the pulse length $L_{f}=M_{1}=M_{0} N$.

For example, if we assume $M=1024$ subchannels, and we choose $M_{0}=2$ and $N_{0}=3$ (which implies $N=1536$ ), and a pulse with length $L_{f}=M_{0} N=3072$, the orthogonality relations will yield 512 submatrices with 2 variables each. In turn, this implies that we need to independently solve 512 subsystems with only 2 variables each.

9.1. Simplified Optimization for Large Number of Subchannels. The FB design procedure is based on the parametrization of the pulse coefficients with angles such that we fulfill the orthogonality conditions, and we deal with a minimal set of free variables [26]. Then, the next step is to find the set of angles such that a certain metric is satisfied (in our case, we use (64) or (65)). The problem becomes complex as the number of subchannels $M$ increases since the number of variables becomes large. For instance, with $M=1024, M_{0}=2, N_{0}=3$, and $L_{f}=2 N$, the amount of angles is 1024. Consequently, it becomes difficult to obtain an acceptable solution with standard methods of optimization, for example, the conjugate gradient method.

To simplify the problem, in [8] it has been proposed to reduce the number of angles by polynomial fitting of them. However, this procedure does not allow obtaining a perfectly orthogonal FB solution. We instead propose an alternative method that significantly simplifies the orthogonal FB design for arbitrarily large $M$ and it maintains the perfect orthogonality. The procedure is iterative and comprises two steps. In the first step, we design the pulse for a value of $M$ that allows using conventional optimization methods, that is, for a number of subchannels that yields a manageable number of variables. In a second iterative step, we increase the number of subchannels by a factor of two and obtain the pulses via interpolation and adjustment of the coefficients such that orthogonality is granted. The procedure is detailed in the following.

Step 1. We minimize for instance the metric (65) for the case $M=M_{0}$. This step can be easily performed since the number of variables is small, for example, for $M=M_{0}$ and $L_{f}=N$, the subsystem has only one variable, and the optimal solution is determined. We denote the prototype pulse obtained at this step with $\hat{g}_{M_{0}}$ where the subscript denotes that the filter is designed for the system with $M_{0}$ channels.

Step 2. We interpolate $\widehat{g}_{M_{0}}$ by a factor 2 with a low-pass filter and we obtain the filter $\hat{h}_{2 M_{0}}=\ell_{2}\left[\hat{g}_{M_{0}}\right]$ which has the same spectrum of $\hat{g}_{M_{0}}$. The preliminary pulse $\hat{h}_{2 M_{0}}$ has the length 
required for the FB with $M=2 M_{0}$ and $L_{f}=N$, that is, for the system with double the number of subchannels of the one at Step 1. We note that the even coefficients of $\hat{h}_{2 M_{0}}$ are the coefficients of $\hat{g}_{M_{0}}(n)$ and the odd coefficients are those derived from interpolation. The filter $\hat{h}_{2 M_{0}}$ is not orthogonal for the system $M=2 M_{0}$ but starting from it we can obtain an orthogonal filter with similar frequency response. The $\mathrm{FB}$ of size $2 M_{0}$ has a system matrix of size that is double that of the FB of size $M_{0}$. The system matrix is block diagonal, and the even coefficients of the pulse $\hat{h}_{2 M_{0}}$ already grant half of the matrix subblocks to be orthogonal. The odd coefficients do not grant the remaining subblocks to be orthogonal. To achieve orthogonality, we keep some of the odd coefficients identical to those obtained via interpolation while we adjust the remaining odd coefficients by solving the set of orthogonality conditions associated to the subblock. We denote this new filter as $\hat{g}_{2 M_{0}}(n)$. Its spectrum is very close to that of the filter $\hat{h}_{2 M_{0}}$. Furthermore, the FB with this new filter is orthogonal.

The procedure at Step 2 can be iteratively repeated starting from $\hat{g}_{2 M_{0}}(n)$ such that we can easily design FBs with prototype pulses $\hat{g}_{2^{k} M_{0}}(n)$ for every $k \in \mathbb{N}$, that is, arbitrarily large number of subchannels.

9.2. Design of Minimum Length FMT Prototype Pulses. The design of minimal length pulses is very important because it allows minimizing the FMT system realization complexity. In this section, we discuss the design of pulses of lengthes $L_{f}=N$ and $L_{f}=2 N$ and we report a detailed description of their construction. We use the least square metric with a root-raised cosine target frequency response. The optimization procedure is the one described in the previous section.

In the following, to simplify the notation, we define the prototype pulse coefficients as

$$
g(n)=h^{*}(-n)=p_{n}
$$

9.2.1. Example of Prototype Pulse Design $\left(M_{0}=2, N_{0}=3\right.$, and $\left.L_{f}=\{N, 2 N\}\right)$. Let us assume a transmission system with $M$ being a power of $2, M_{0}=2, N_{0}=3$, and $L_{f}=2 N$. Hence, $N=3 M / 2$. The submatrices in (63) have the following structure:

$$
\mathbf{G}_{a}(z)=\left[\begin{array}{ccc}
p_{a} & p_{M+a} & p_{2 M+a} \\
z^{-1} p_{M_{1}+(a-N)} & z^{-1} p_{M_{1}+(M+a-N)} & p_{2 M+a-N}
\end{array}\right]^{T},
$$

where according to (66) $p_{n}$ are the coefficients of the prototype pulse and $a \in\left\{0,1, \ldots, M / M_{0}-1\right\}$. If a filter coefficient is present in the submatrix $\mathbf{G}_{i}$, it cannot be present in any other submatrix $\mathbf{G}_{j}$ with $j \neq i$. This implies that the subsystems are uncoupled.

From (67), we can derive the orthogonal conditions also for $L_{f}=N$ simply setting certain elements to zero, as detailed in the following.
Case $1\left(L_{f}=N\right)$. For the case $L_{f}=N$, the orthogonality conditions for the ath subsystem are given by the following equations:

$$
\begin{gathered}
p_{a}^{2}+p_{M+a}^{2}=1, \\
p_{2 M+a-N}^{2}=1
\end{gathered}
$$

with $a \in\left\{0,1, \ldots, M / M_{0}-1\right\}$. In order to solve the system using a minimal set of variables, we can parameterize the pulse coefficients with angles as follows:

$$
\begin{gathered}
p_{a}=\cos \left(\theta_{a, 1}\right), \\
p_{M+a}=\sin \left(\theta_{a, 1}\right), \\
p_{2 M+a-N}=1 .
\end{gathered}
$$

Case $2\left(L_{f}=2 N\right)$. Now, the orthogonality conditions for the ath subsystem are given by the following equations:

$$
\begin{gathered}
p_{a}^{2}+p_{M+a}^{2}+p_{2 M+a}^{2}=1, \\
p_{M_{1}+(a-N)}^{2}+p_{M_{1}+(M+a-N)}^{2}+p_{2 M+a-N}^{2}=1 \\
p_{a} p_{M_{1}+(a-N)}+p_{M+a} p_{M_{1}+(M+a-N)}=0 \\
p_{2 M+a} p_{2 M+a-N}=0
\end{gathered}
$$

with $a \in\left\{0,1, \ldots, M / M_{0}-1\right\}$. Choosing $p_{2 M+a}=0$, and parameterizing the pulse coefficients with angles, the system solution is

$$
\begin{gathered}
p_{a}=\cos \left(\theta_{a, 1}\right), \\
p_{M+a}=\sin \left(\theta_{a, 1}\right), \\
p_{2 M+a}=0, \\
p_{2 M+a-N}=\cos \left(\theta_{a, 2}\right), \\
p_{M_{1}+(a-N)}=-\sin \left(\theta_{a, 1}\right) \sin \left(\theta_{a, 2}\right), \\
p_{M_{1}+(M+a-N)}=\cos \left(\theta_{a, 1}\right) \sin \left(\theta_{a, 2}\right) .
\end{gathered}
$$

For every choice of $\left(\theta_{a, 1}, \theta_{a, 2}\right)$ with $a \in\left\{0,1, \ldots, M / M_{0}-\right.$ $1\}$, the FMT scheme is orthogonal. We then define the vectors $\boldsymbol{\theta}_{1}=\left[\theta_{0,1}, \theta_{1,1}, \ldots, \theta_{M / M_{0}, 1}\right]$ and $\boldsymbol{\theta}_{2}=\left[\theta_{0,2}, \theta_{1,2}, \ldots, \theta_{M / M_{0}, 2}\right]$ and we search for pulses that satisfy the metrices (64) and (65). The search is done according to the algorithm described in Section 9.1. In Figures 8 and 9, we show the obtained pulses for $M=64,256,1024$.

9.2.2. Example of Prototype Pulse Design $\left(M_{0}=4, N_{0}=5\right.$, and $\left.L_{f}=\left\{N, 2 N, \ldots, M_{0} N\right\}\right)$. For the case, $M_{0}=4, N_{0}=5$, and $L_{f}=M_{0} N$, the submatrices have the following structure:

$$
\begin{aligned}
& \mathbf{G}_{a}= \\
& {\left[\begin{array}{ccccc}
p_{a} & p_{M+a} & p_{2 M+a} & p_{3 M+a} & p_{4 M+a} \\
z^{-1} p_{M_{1}+(a-N)} & \mathfrak{A} & p_{2 M+a-N} & \mathfrak{B} & p_{4 M+a-N} \\
z^{-1} p_{M_{1}+(a-2 N)} & \mathfrak{C} & z^{-1} p_{M_{1}+(2 M+a-2 N)} & \mathfrak{D} & p_{4 M+a-2 N} \\
z^{-1} p_{M_{1}+(a-3 N)} & \mathfrak{Z} & z^{-1} p_{M_{1}+(2 M+a-3 N)} & \mathfrak{F} & p_{4 M+a-3 N}
\end{array}\right]^{T},}
\end{aligned}
$$




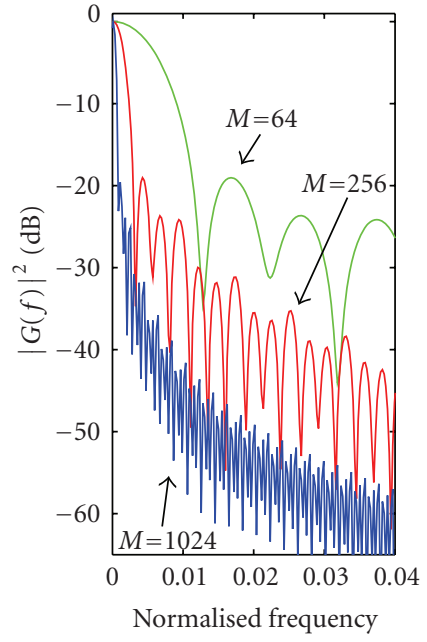

(a)

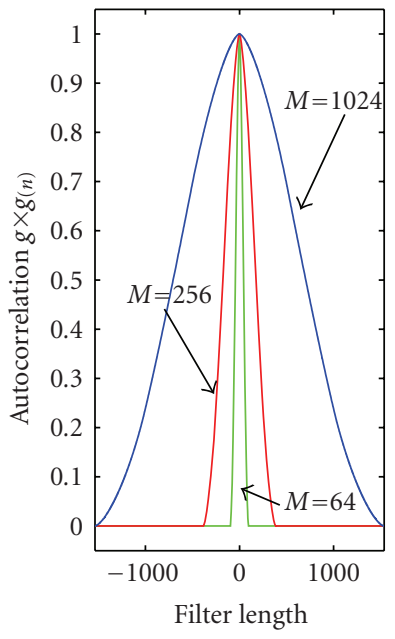

(b)
FIGURE 8: Frequency response and pulse autocorrelation for parameters $M_{0}=2, N_{0}=3$, and $L_{f}=N$.

where $\mathfrak{A}$ denotes $z^{-1} p_{M_{1}+(M+a-N)}, \mathfrak{B}$ dentoes $p_{3 M+a-N}, \mathfrak{C}$ denotes $z^{-1} p_{M_{1}+(M+a-2 N)}, \mathfrak{D}$ denotes $p_{3 M+a-2 N}, \mathfrak{Z}$ denotes $z^{-1} p_{M_{1}+(M+a-3 N)}$, and $\mathfrak{F}$ denotes $z^{-1} p_{M_{1}+(3 M+a-3 N)}$.

Equation (72) can be used to derive the orthogonality conditions for the cases $L_{f}<M_{0} N$ simply setting certain elements to zero, as detailed in following.

Case $1\left(L_{f}=N\right)$. Setting $p_{i}=0$ with $i \in\{6, \ldots, 19\}$, the orthogonality condition for the ath subsystem yields the following equations:

$$
\begin{gathered}
p_{a}^{2}+p_{M+a}^{2}=1, \\
p_{2 M+a-N}^{2}=1, \\
p_{3 M+a-2 N}^{2}=1, \\
p_{4 M+a-3 N}^{2}=1 .
\end{gathered}
$$
yields

The system can be parameterized with angles which

$$
\begin{gathered}
p_{a}=\cos \left(\theta_{a, 1}\right), \\
p_{M+a}=\sin \left(\theta_{a, 1}\right), \\
p_{2 M+a-N}^{2}=1, \\
p_{3 M+a-2 N}^{2}=1, \\
p_{4 M+a-3 N}^{2}=1 .
\end{gathered}
$$

Case $2\left(L_{f}=2 N\right)$. Setting $p_{i}=0$ with $i \in\{11, \ldots, 19\}$, the orthogonality property for the ath subsystem is determined by the following equations:

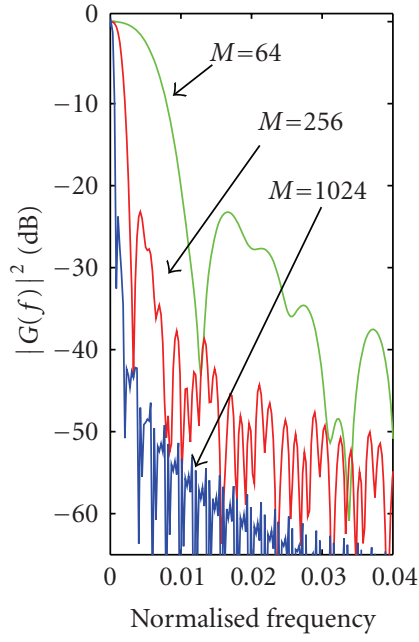

(a)

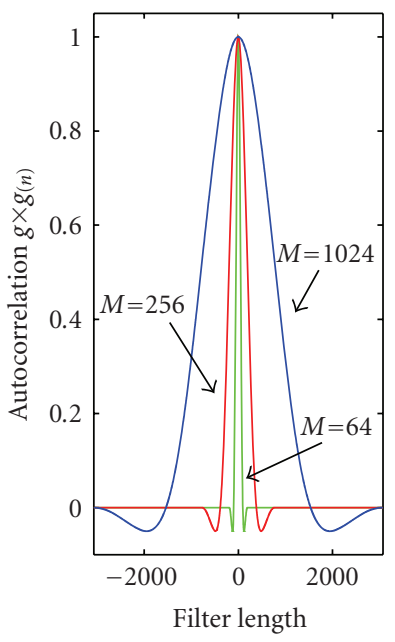

(b)
Figure 9: Frequency response and pulse autocorrelation for parameters $M_{0}=2, N_{0}=3$, and $L_{f}=2 N$.

$$
\begin{gathered}
p_{a}^{2}+p_{M+a}^{2}+p_{2 M+a}^{2}=1, \\
p_{2 M+a-N}^{2}+p_{3 M+a-N}^{2}=1, \\
p_{M_{1}+(a-2 N)}^{2}+p_{3 M+a-2 N}^{2}+p_{4 M+a-2 N}^{2}=1, \\
p_{M_{1}+(a-3 N)}^{2}+p_{M_{1}+(M+a-3 N)}^{2}+p_{4 M+a-3 N}^{2}=1, \\
p_{a} p_{M_{1}+(a-3 N)}+p_{M+a} p_{M_{1}+(M+a-3 N)}=0, \\
p_{a} p_{M_{1}+(a-2 N)}=0, \\
p_{2 M+a-N}+p_{2 M+a}=0, \\
p_{3 M+a-2 N} p_{3 M+a-N}=0, \\
p_{4 M+a-3 N} p_{4 M+a-2 N}=0 .
\end{gathered}
$$

Setting $p_{M_{1}+(M+a-3 N)}=p_{3 M+a-2 N}=p_{2 M+a}=p_{M_{1}+(a-2 N)}=0$ we obtain

$$
\begin{gathered}
p_{a}=\cos \left(\theta_{a, 1}\right), \\
p_{M+a}=\sin \left(\theta_{a, 1}\right), \\
p_{2 M+a-N}=\cos \left(\theta_{a, 2}\right), \\
p_{3 M+a-N}=\sin \left(\theta_{a, 2}\right), \\
p_{4 M+a-2 N}=1, \\
p_{M_{1}+(a-3 N)}=\sin \left(\theta_{a, 1}\right), \\
p_{M_{1}+(M+a-3 N)}=-\cos \left(\theta_{a, 1}\right) .
\end{gathered}
$$

In Figures 10 and 11, we show the obtained pulses for $M=64,256,1024$.

9.2.3. Generic Design for $M_{0}=2^{n}, N_{0}=M_{0}+1$, and $L_{f}=N$. The previous examples can be generalized to the case given by the parameters $M_{0}=2^{n}, N_{0}=M_{0}+1$, and 


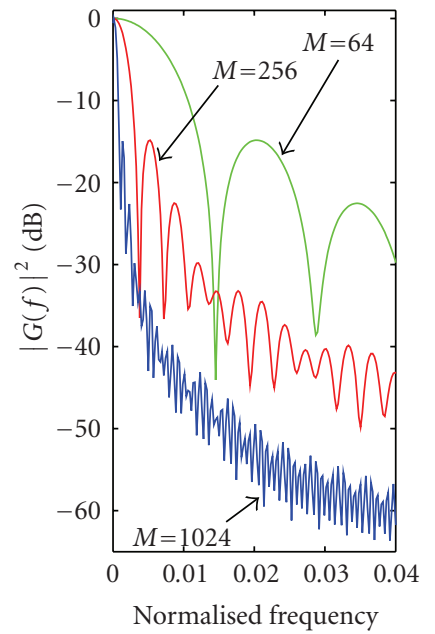

(a)

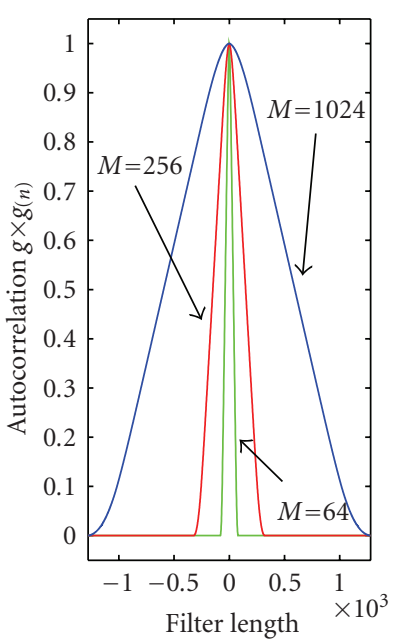

(b)

Figure 10: Frequency response and pulse autocorrelation for parameters $M_{0}=4, N_{0}=5$, and $L_{f}=N$.

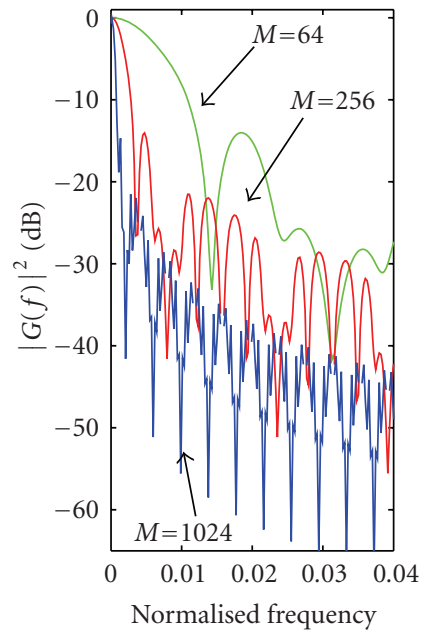

(a)

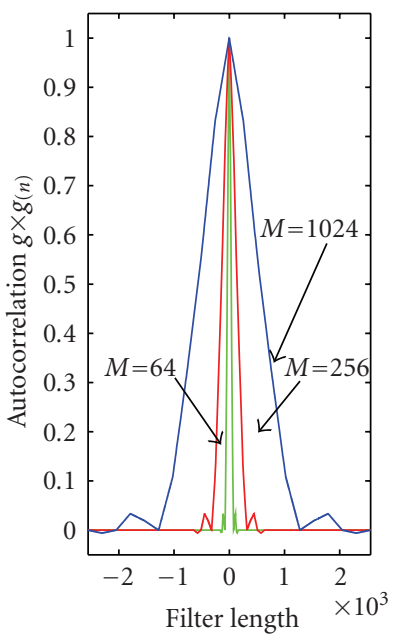

(b)

Figure 11: Frequency response and pulse autocorrelation for parameters $M_{0}=4, N_{0}=5$, and $L_{f}=2 N$.

$L_{f}=N$. To do so we simply set $p_{i}=0$ for $i>N_{0}$, then the orthogonality conditions for the ath subsystem yield the following equations:

$$
\begin{gathered}
p_{a}^{2}+p_{M+a}^{2}=1, \\
p_{(k+1) M+a-k N}^{2}=1, \quad k \in\left\{1, \ldots, M_{0}-1\right\} .
\end{gathered}
$$

The system can be parameterized with angles which yields

$$
\begin{gathered}
p_{a}=\cos \left(\theta_{a, 1}\right), \\
p_{M+a}=\sin \left(\theta_{a, 1}\right), \\
p_{(k+1) M+a-k N}^{2}=1, \quad k \in\left\{1, \ldots, M_{0}-1\right\} .
\end{gathered}
$$

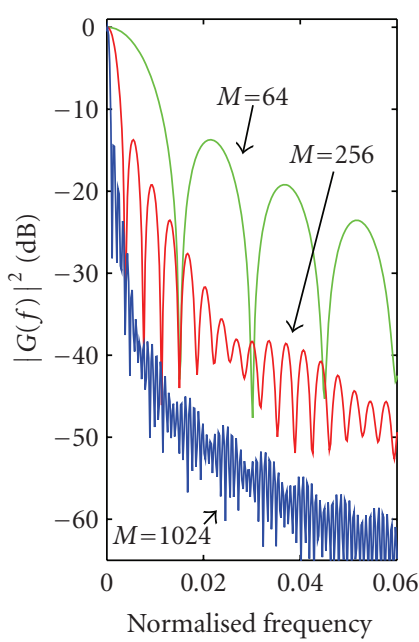

(a)

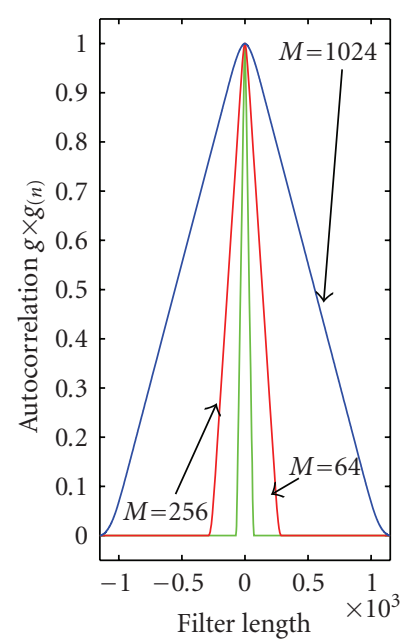

(b)
Figure 12: Frequency response and pulse autocorrelation for parameters $M_{0}=8, N_{0}=9$, and $L_{f}=N$.

As an example, in Figure 12 we show the obtained pulses for $M=64,256,1024, M_{0}=8, N_{0}=9$, and $L_{f}=N$.

\section{Performance in Wireless Fading Channels}

In order to evaluate the robustness of the FMT scheme with the proposed pulses, we first consider transmission over a wireless dispersive fading channel having impulse response $g_{\mathrm{ch}}(n)=\sum_{p=0}^{N_{p}-1} \alpha_{p} \delta(n-p)$, where $\alpha_{p}$ are independent circular symmetric complex Gaussian variables with power $\Omega_{p}=\Omega_{0} e^{-p / \gamma}$, and $\gamma$ is the normalized delay spread. The channel is truncated at $-20 \mathrm{~dB}$ and normalized to have unit average energy. This channel introduces a loss of system orthogonality that we evaluate in terms of expected Signal-to-Interference Power Ratio SIR, versus delay spread $\gamma$. The SIR is evaluated as follows: first we compute the subchannel signal-to-interference (ISI plus ICI) power ratio for a given channel realization. Then, we compute the average signal-to-interference ratio averaged over the subchannels. Finally, we evaluate the expected (averaged over the channel realizations) signal-to-interference power ratio.

The simulation has been done for the case $M=$ $64,256,1024, L_{f}=N$, and $N=3 / 2 M$ in Figure $13, N=$ $5 / 4 M$ in Figure 14, $N=9 / 8 M$ in Figure 15. To benchmark the performance of the proposed pulses, we consider the case $M=64$ subcarriers with a Gaussian/IOTA pulse of length $L_{f}=N$ which is a truncated version of the Gaussian/IOTA pulse family presented in [12] that is known to be optimally time-frequency localized. We furthermore consider a conventional root-raised cosine (rrc) pulse with roll-of factor 0.2 and variable length $L_{f}=2 \mathrm{~N}, 6 \mathrm{~N}, 20 \mathrm{~N}$.

Figures 13-15 show that the FMT system, in the considered channel, has considerable better performance with the orthogonal pulses than with the rrc pulse of length $2 N$, and the Gaussian/IOTA pulse of length $N$ especially for low values of delay spread. For very high delay spreads, 


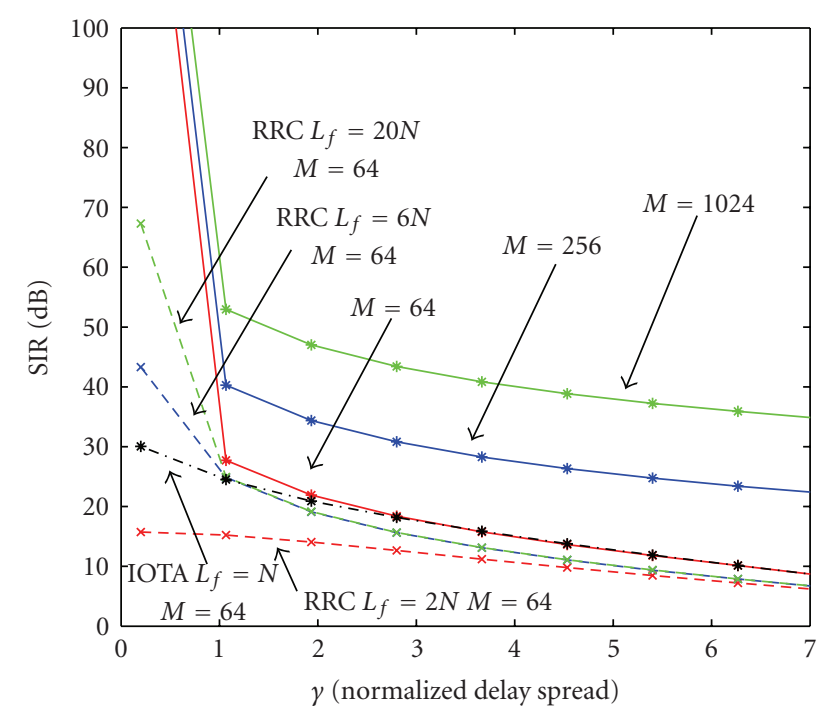

FIGURE 13: SIR versus the normalized delay spread $\gamma$ for orthogonal pulse (solid lines) with $M_{0}=2, N_{0}=3$, and $L_{f}=N$.

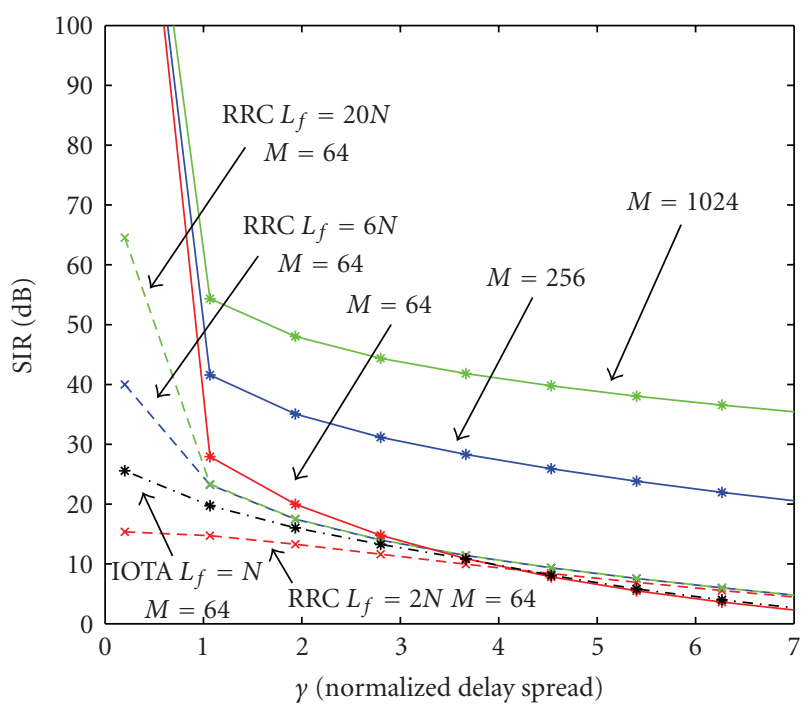

FIGURE 14: SIR versus the normalized delay spread $\gamma$ for orthogonal pulse (solid lines) with $M_{0}=4, N_{0}=5$, and $L_{f}=N$.

the Gaussian/IOTA pulse yields similar performance to the orthogonal pulse because of the distortions introduced by the frequency selective channel. The rrc pulse will have a better behavior only if it has large length $(6 \mathrm{~N}$ and $20 \mathrm{~N}$, in the figures) which however increases the complexity of the realization.

Furthermore, the figures show that better SIR is obtained by increasing the number of carriers since the subchannels exhibit a flatter frequency response.

Now, we consider the application of FMT in indoor wireless LAN channels. We have used the IEEE 802.11 channel model presented in [23]. This model generates channels belonging to five classes labeled with B,C,D,E,F. Each class is a representative of a certain environment,

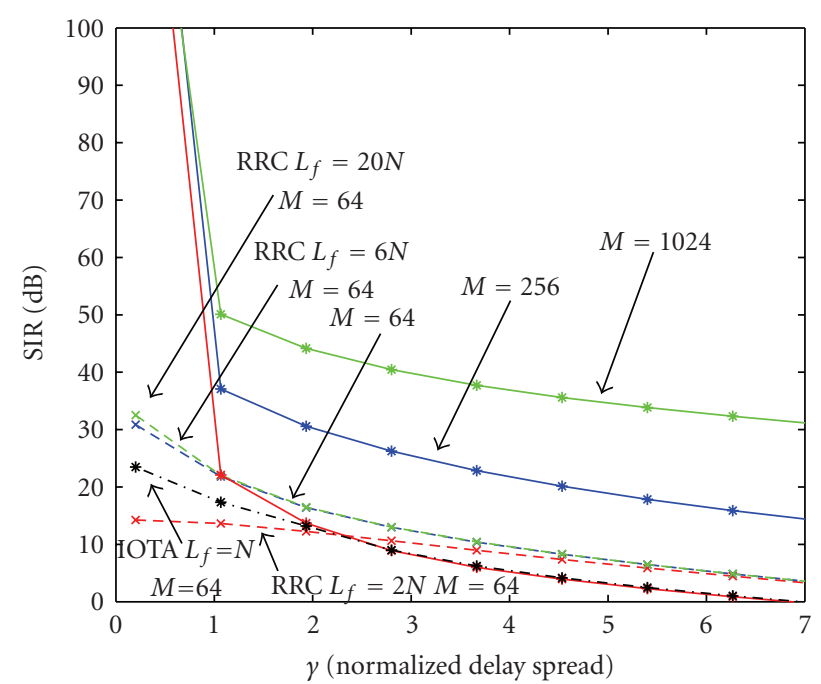

FIGURE 15: SIR versus the normalized delay spread $\gamma$ for orthogonal pulse with (solid lines) $M_{0}=8, N_{0}=9$, and $L_{f}=N$.

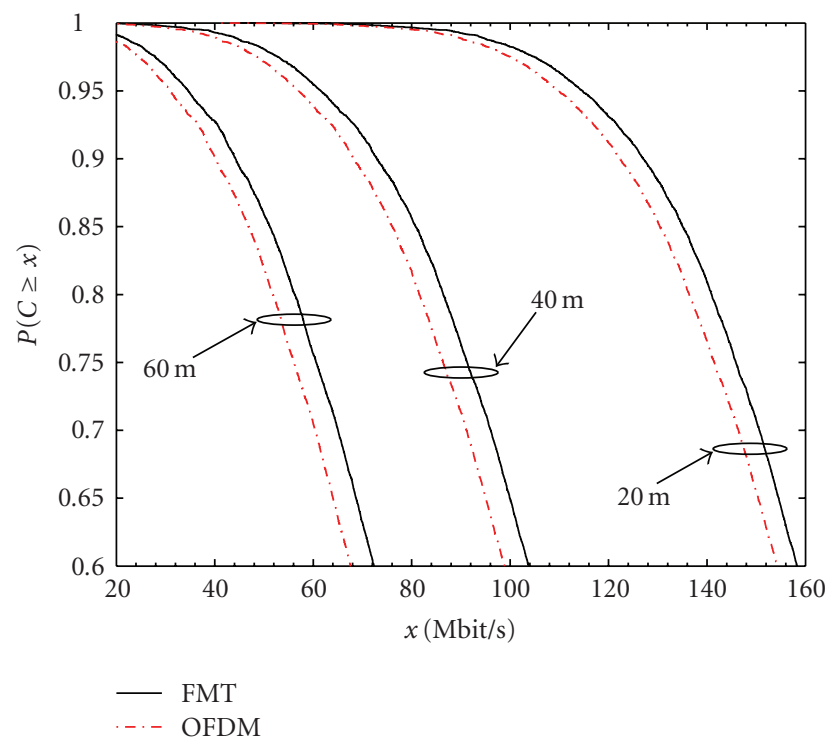

FIGURE 16: Capacity of the FMT with $M=64, N=80$, and $L_{f}=80$ versus $\mathrm{OFDM}$ with $M=64$, and $C P=16$ samples.

for example, small office, large open space/office with line of sight (LOS), and non-LOS (NLOS) propagation, and so on. Both small scale multipath fading and large scale path loss fading as a function of distance are taken into account. Doppler effects from movement are also considered. Although the model allows considering MIMO channels, we restrict ourselves to the case of single-transmit/single-receive antenna. For a detailed description of the model, see [23] and references therein. The continuous time complex impulse response provided by such a model (with slow fading) can be written as

$$
\widehat{g}_{\mathrm{ch}}(n, d)=A(d) \sum_{p=0}^{\nu-1} \beta(p) \delta\left(n-\tau_{p}\right),
$$


where $A(d)$ is the attenuation from path-loss when the transmitter and the receiver are at distance $d, \beta(p)$ is the tap amplitude, and $\tau_{p}$ is the time delay. The number of multipath components is denoted with $\nu$. We consider class $B$ that assumes a LOS environment. The first tap has Rice amplitude, while the remaining $v-1$ taps are Rayleigh distributed. To obtain the equivalent discrete time channel impulse response, we filter the channel response with a lowpass pulse, and we sample the outputs at rate $1 / T$ that is equal to the transmission bandwidth of $20 \mathrm{MHz}$.

Now, we evaluate the system capacity with the above channel model assuming parallel Gaussian channels. That is, we assume additive white Gaussian noise, and independent and Gaussian distributed input signals, which renders ISI and ICI also Gaussian. Furthermore, we use single tap subchannel equalization, that is, no attempt is made to suppress ISI and ICI. Then, the maximal data rate, for a given channel realization, is

$$
C=\frac{1}{N T} \sum_{k=0}^{M-1} \log _{2}\left(1+\operatorname{SINR}^{(k)}\right)[\mathrm{bit} / \mathrm{s}] .
$$

In (80), $\operatorname{SINR}^{(k)}$ denotes the signal over interference plus noise ratio experienced in subchannel $k$ for a given channel realization.

In Figure 16, we show the complementary cumulative distribution function of the capacity (80). The results have been obtained assuming the class B channel with distances between transmitter and receiver equal to $20 \mathrm{~m}, 40 \mathrm{~m}$, and $60 \mathrm{~m}$. The FMT system uses the pulse in Figure 10 with $M=64$ subchannels, and single tap equalization. As a comparison, we also report the performance of OFDM with $M=64$ subchannels and a cyclic prefix of length 16 samples $(0.8 \mu \mathrm{s})$ as defined in the IEEE 802.11 standard. The two systems have identical overhead. We assume a transmitted power spectra density (PSD) of $-53 \mathrm{dBm} / \mathrm{Hz}$ and a noise PSD of $-168 \mathrm{dBm} / \mathrm{Hz}$.

The results show that in the considered scenario, FMT significantly outperforms OFDM yet having similar complexity since single tap equalization is used in both systems, and the pulse used in FMT has minimal length. For instance, for $80 \%$ of channel realizations, OFDM exceeds $81 \mathrm{Mbits} / \mathrm{s}$ at $40 \mathrm{~m}$ while FMT exceeds $86 \mathrm{Mbits} / \mathrm{s}$. Performance improvements are expected if more powerful subchannel equalization is deployed in FMT.

\section{Conclusions}

In this paper, we have compared three efficient realizations of a filtered multitone (FMT) modulation system. We have shown that these implementations have the same complexity in terms of complex operations, and similar memory requirements, but they are different in terms of hardware implementation and matrix representation due to a different polyphase FB structure.

We have then considered the design of an orthogonal FMT system exploiting the matrix structure of the first realization (the method in [6]) that allows deriving a method that considerablly simplifies the design of DFT modulated orthogonal filter banks for certain choices of the parameters and for arbitrarily large number of subchannels.

Several examples of pulses with minimal length have been reported and the performance of the system in typical wireless multi-path fading channels has been shown. The comparison with the conventional truncated root-raisedcosine pulse and the Gaussian/IOTA pulse has shown that the proposed filter design yields significant improved robustness to multi-path fading. Furthermore, the comparison with cyclically prefixed OFDM using the IEEE 802.11 WLAN class B channel has shown that FMT outperforms OFDM, yet having similar complexity.

\section{Appendices}

\section{A. Efficient Implementation Using Cvetković and Vetterli Method}

In this appendix, we derive the Method B proposed by Cvetković and Vetterli in [7], and more recently by Siclet et al. in [8] and by McGee in [9].

A.1. Synthesis Bank (Method B). The subchannel pulse $g^{(k)}$ can be obtained from its A-type $M_{1}$ order polyphase decomposition as follows:

$$
\begin{gathered}
g^{(k)}(n)=\sum_{i=0}^{M_{1}-1} \tau^{-i}\left[\ell_{M_{1}}\left[g_{i}^{(k)}\right]\right](n), \\
g_{i}^{(k)}\left(M_{1} n\right)=g\left(M_{1} n+i\right) W_{M}^{-k\left(M_{1} n+i\right)}=g_{i}\left(M_{1} n\right) W_{M}^{-k i},
\end{gathered}
$$

where $g_{i}$ is the $i$ th polyphase decomposition of order $M_{1}$ of the synthesis prototype pulse.

Using this result, the signal at the synthesis FB (21) can be written as

$$
\begin{aligned}
x & =\sum_{k=0}^{M-1}\left[\ell_{N}\left[a^{(k)}\right] * \sum_{i=0}^{M_{1}-1} \tau^{-i}\left[\ell_{M_{1}}\left[g_{i}\right]\right] W_{M}^{-k i}\right] \\
& =\sum_{i=0}^{M_{1}-1}\left[\ell_{N}\left[\sum_{k=0}^{M-1} a^{(k)} W_{M}^{-k i}\right] * \tau^{-i}\left[\ell_{M_{1}}\left[g_{i}\right]\right]\right] .
\end{aligned}
$$

Let us define $A^{(i)}$ as the $M$-point IDFT of the signal $a^{(k)}$. Then, since $M_{1}=M_{0} N$, we obtain

$$
\begin{aligned}
x & =\sum_{i=0}^{M_{1}-1}\left[\ell_{N}\left[A^{(i)}\right] * \tau^{-i}\left[\ell_{M_{0} N}\left[g_{i}\right]\right]\right] \\
& =\sum_{i=0}^{M_{1}-1} \tau^{-i}\left[\ell_{N}\left[A^{(i)}\right] * \ell_{M_{0} N}\left[g_{i}\right]\right] \quad(\text { using (15)) } \\
& \left.=\sum_{i=0}^{M_{1}-1} \tau^{-i}\left[\ell_{N}\left[A^{(i)} * \ell_{M_{0}}\left[g_{i}\right]\right]\right] \quad \text { (A. } 4\right)
\end{aligned}
$$

We now define the indices

$$
i=p+M m=\alpha+N \beta
$$


with $m \in\left\{0,1, \ldots, N_{0}-1\right\}, p \in\{0,1, \ldots, M-1\}, \alpha \in$ $\{0,1, \ldots, N-1\}, \beta \in\left\{0,1, \ldots, M_{0}-1\right\}$, and $p=\bmod [a+$ $N \beta, M]$ and $m=\operatorname{div}[a+N \beta, M]$.

Finally, we can rearrange (A.5) as follows:

$$
x=\sum_{\alpha=0}^{N-1 M_{0}-1} \sum_{\beta=0}^{-\alpha-N \beta}\left[\ell_{N}\left[A^{(\bmod [\alpha+N \beta, M])} * \ell_{M_{0}}\left[g_{(\alpha+N \beta)}\right]\right]\right]
$$

$$
=\sum_{\alpha=0}^{N-1} \sum_{\beta=0}^{M_{0}-1} \tau^{-\alpha}\left[\ell_{N}\left[A^{(\bmod [\alpha+N \beta, M])} * \tau^{-\beta} \ell_{M_{0}}\left[g_{(\alpha+N \beta)}\right]\right]\right]
$$

(using (6), (14), and (15))

$$
=\sum_{\alpha=0}^{N-1} \tau^{-\alpha} \ell_{N}\left[\sum_{\beta=0}^{M_{0}-1} A^{(\bmod [\alpha+N \beta, M])} * \tau^{-\beta} \ell_{M_{0}}\left[g_{(\alpha+N \beta)}\right]\right]
$$

According to (A.9), the transmitted signal is obtained by a S/P conversion of order $N$ of the polyphase components (41).

A.2. Analysis Bank (Method B). To start we can express the $k$ th subchannel filter $h^{(k)}$ in the analysis FB (22) from its Btype $M_{1}$ order polyphase components $h_{-l}^{(k)}: \mathbb{Z}\left(M_{1}\right): \rightarrow \mathbb{C}$ as follows:

$$
\begin{gathered}
h^{(k)}(n)=\sum_{l=0}^{M_{1}-1} \tau^{l}\left[\ell_{M_{1}}\left[h_{-l}^{(k)}\right]\right](n), \\
h_{-l}^{(k)}=h\left(M_{1} n-l\right) W_{M}^{-k\left(M_{1} n-l\right)}=h_{-l}\left(M_{1} n\right) W_{M}^{k l},
\end{gathered}
$$

where $h_{-l}$ is the $l$ th polyphase component of order $M_{1}$ of the analysis prototype pulse.

Then, the FB outputs (22) can be obtained as follows:

$$
\begin{aligned}
b^{(k)} & =\mathcal{C}_{N}\left[x * \sum_{l=0}^{M_{1}-1} \tau^{l}\left[\ell_{M_{1}}\left[h_{-l}\right]\right] W_{M}^{k l}\right] \\
& =\sum_{l=0}^{M_{1}-1} \mathcal{C}_{N}\left[\tau^{l}[x] * \ell_{M_{0} N}\left[h_{-l}\right]\right] W_{M}^{k l} \\
\quad(\text { using }(7) \text { and }(15)) & (\text { A.12) } \\
= & \sum_{l=0}^{M_{1}-1} \mathcal{C}_{N}\left[\tau^{l}[x]\right] * \ell_{M_{0}}\left[h_{-l}\right] W_{M}^{k l} \quad(\text { using (12) and (17)). }
\end{aligned}
$$

Now, we redefine the indexes $l=p+M m=\alpha+$ $N \beta$ with $m \in\left\{0,1, \ldots, N_{0}-1\right\}, p \in\{0,1, \ldots, M-$ $1\}, \alpha \in\{0,1, \ldots, N-1\}, \beta \in\left\{0,1, \ldots, M_{0}-1\right\}$, and $\alpha=\bmod [p+M m, N], \beta=\operatorname{div}[p+M m, N]$. Thus, we obtain

$$
\begin{aligned}
b^{(k)} & =\sum_{p=0}^{M-1} \sum_{m=0}^{N_{0}-1}\left[\mathcal{C}_{N}\left[\tau^{\alpha+N \beta}[x]\right] * \ell_{M_{0}}\left[h_{-p-M m}\right]\right] W_{M}^{k p} \\
& =\sum_{p=0}^{M-1} \sum_{m=0}^{N_{0}-1}\left[\tau^{\beta}\left[\mathcal{C}_{N}\left[\tau^{\alpha}[x]\right] * \ell_{M_{0}}\left[h_{-p-M m}\right]\right]\right] W_{M}^{k p}
\end{aligned}
$$

(using (10)).

Since $\mathcal{C}_{N}\left[\tau^{\alpha}[x]\right]$ is the $\alpha$ th polyphase component of $x$, we can define it as follows:

$$
x_{\alpha}=\mathcal{C}_{N}\left[\tau^{\alpha}[x]\right]
$$

Substituting $x_{\alpha}, \alpha$ and $\beta$, we obtain the realization of the analysis $\mathrm{FB}$ as in (44):

$b^{(k)}=$

$$
\sum_{p=0}^{M-1} \sum_{m=0}^{N_{0}-1}\left[\tau^{\operatorname{div}[p+M m, N]}\left[x_{\bmod [p+M m, N]} * \ell_{M_{0}}\left[h_{-p-M m}\right]\right]\right] W_{M}^{k p}
$$

\section{B. Efficient Implementation Using Weiss and Stewart Method}

In this appendix, we derive the Method $\mathrm{C}$ proposed by Weiss and Stewart in [10].

B.1. Synthesis Bank (Method C). Similarly to the derivation for Method B in Appendix A, we can express the subchannel pulse $g^{(k)}$ starting from its A-type $L_{f}=L_{M} N=L_{N} M$ order polyphase components where $L_{f}$ is the pulse length. Then, the transmitted signal can be written as

$$
x=\sum_{i=0}^{L_{f}-1} \tau^{-i}\left[\ell_{N}\left[A^{(i)} * \ell_{L_{M}}\left[g_{i}\right]\right]\right] .
$$

Since the polyphase decomposition of the prototype pulse yields length one components, we can write them as

$$
g_{i}\left(L_{f} n\right)=g(i) \times \delta\left(L_{f} n\right),
$$

where $\delta\left(L_{f} n\right)$ is the Dirac delta function.

Therefore, we obtain

$$
x=\sum_{i=0}^{L_{f}-1} \tau^{-i}\left[\ell_{N}\left[A^{(i)} \times g(i)\right]\right] .
$$

We now redefine the indices $i=\alpha+N \beta=p+M m$ with $\alpha \in\{0,1, \ldots, N-1\}, \beta \in\left\{0,1, \ldots, M_{0}-1\right\}, m \in$ $\left\{0,1, \ldots, N_{0}-1\right\}, p \in\{0,1, \ldots, M-1\}$, and $p=\bmod [\alpha+$ $N \beta, M], m=\operatorname{div}[\alpha+N \beta, M]$. Finally, we reach to the 
following final relation:

$$
\begin{aligned}
x & =\sum_{\alpha=0}^{N-1} \sum_{\beta=0}^{L_{M}-1} \tau^{-\alpha-N \beta}\left[\ell_{N}\left[A^{(p+M m)} \times g(\alpha+N \beta)\right]\right] \\
& =\sum_{\alpha=0}^{N-1} \tau^{-\alpha}\left[\ell_{N}\left[\sum_{\beta=0}^{L_{M}-1} \tau^{-\beta}\left[A^{(\bmod [\alpha+N \beta, M])} \times g(\alpha+N \beta)\right]\right]\right]
\end{aligned}
$$

(using (10) and (11))

which shows that the transmitted signal is obtained by an $\mathrm{S} / \mathrm{P}$ conversion of the polyphase components in (47).

B.2. Analysis Bank (Method C). We can perform a B-type $L_{f}=L_{M} N=L_{N} M$ order polyphase decomposition on the analysis subchannel pulse $h^{(k)}$ similarly to what is done in the synthesis stage. We assume without loss of generality the pulse $h(n)$ to be anticausal and defined for $n \in\left\{-L_{f}+1, \ldots, 0\right\}$. Since the polyphase decomposition of the prototype pulse yields length one components, we can write them as

$$
h_{-i}(n)=h(-i) \times \delta\left(L_{f} n\right) .
$$

Thus, the analysis FB outputs are obtained as follows:

$$
\begin{aligned}
b^{(k)}=\sum_{p=0}^{M-1}\left(\sum_{m=0}^{L_{N}-1} \tau^{\operatorname{div}[p+M m, N]}[\right. & x_{\bmod [p+M m, N]} \\
& \left.\left.\times h\left(L_{f}-p-M m\right)\right]\right) W_{M}^{k p}
\end{aligned}
$$

which proves (48).

\section{Acknowledgment}

The work of this paper has been partially supported by the European Community Seventh Framework Programme FP7/2007-2013 under Grant agreement no. 213311, project OMEGA-Home Gigabit Networks.

\section{References}

[1] M. L. Doelz, E. T. Heald, and D. L. Martin, "Binary data transmission techniques for linear systems," Proceedings of the IRE, pp. 656-661, 1957.

[2] J. A. C. Bingham, "Multicarrier modulation for data transmission: an idea whose time has come," IEEE Communications Magazine, vol. 28, no. 5, pp. 5-14, 1990.

[3] G. Cherubini, E. Eleftheriou, and S. Olcer, "Filtered multitone modulation for very high-speed digital subscribe lines," IEEE Journal on Selected Areas in Communication, pp. 1016-1028, 2002.

[4] A. M. Tonello, "Asynchronous multicarrier multiple access: optimal and sub-optimal detection and decoding," Bell Labs Technical Journal, vol. 7, no. 3, pp. 191-217, 2003.
[5] A. M. Tonello and F. Pecile, "Efficient architectures for multiuser FMT systems and application to power line communications," IEEE Transaction on Communications, vol. 57, no. 5, pp. 1275-1279, 2009.

[6] A. M. Tonello, "Time domain and frequency domain implementations of FMT modulation architectures," in Proceedings of the IEEE International Conference on Acoustics, Speech, and Signal Processing (ICASSP '06), vol. 4, pp. 625-628, Toulouse, France, May 2006.

[7] Z. Cvetković and M. Vetterli, "Tight Weyl-Heisemberg frames in $\ell^{2}(\mathbb{Z})$," IEEE Transactions on Signal Processing, vol. 46, no. 5, pp. 1256-1259, 1998.

[8] C. Siclet, P. Siohan, and D. Pinchon, "Perfect reconstruction conditions and design of oversampled DFT-modulated transmultiplexers," EURASIP Journal on Applied Signal Processing, vol. 2006, Article ID 15756, 14 pages, 2006.

[9] W. F. McGee, "Efficient Realization of Filtered Multitone Transmitters and Receivers and Oversampled Analysis/Synthesis Filterbank Pairs for Perfect and Non Perfect Reconstruction," Personal correspondence, August 2007.

[10] S. Weiss and R. W. Stewart, "Fast implementation of oversampled modulated filter banks," Electronics Letters, vol. 36, no. 17, pp. 1502-1503, 2000.

[11] T. Hunziker and D. Dahlhaus, "Iterative detection for multicarrier transmission employing time-frequency concentrated pulses," IEEE Transactions on Communications, vol. 51, no. 4, pp. 641-651, 2003.

[12] B. Le Floch, M. Alard, and C. Berrou, "Coded orthogonal frequency division multiplex," Proceedings of the IEEE, vol. 83, no. 6, pp. 982-996, 1995.

[13] B. Le Floch, R. Halbert-Lassalle, and D. Castelain, "Digital sound broadcasting to mobile receivers," IEEE Transactions on Consumer Electronics, vol. 35, no. 3, pp. 493-503, 1989.

[14] R. Haas and J.-C. Belfiore, "A time-frequency well-localized pulse for multiple carrier transmission," Wireless Personal Communications, vol. 5, no. 1, pp. 1-18, 1997.

[15] W. Kozek and A. F. Molisch, "Nonorthogonal pulseshapes for multicarrier communications in doubly dispersive channels," IEEE Journal on Selected Areas in Communications, vol. 16, no. 8, pp. 1579-1589, 1998.

[16] D. Schafhuber, G. Matz, and F. Hlawatsch, "Pulse-shaping OFDM/BFDM systems for time-varying channels: ISI/ICI analysis, optimal pulse design, and efficient implementation," in Proceedings of the The 13th IEEE International Symposium on Personal, Indoor and Mobile Radio Communications (PIMRC '02), vol. 3, pp. 1012-1016, Lisbon, Portugal, September 2002.

[17] T. Strohmer and S. Beaver, "Optimal OFDM design for time-frequency dispersive channels," IEEE Transactions on Communications, vol. 51, no. 7, pp. 1111-1122, 2003.

[18] M. Harteneck, S. Weiss, and W. Stewart, "Design of near perfect reconstruction oversampled filter banks for subband adaptive filters," IEEE Transactions on Circuits and Systems Part II, vol. 46, no. 8, pp. 1081-1086, 1999.

[19] B. Borna and T. N. Davidson, "Efficient design of FMT systems," IEEE Transactions on Communications, vol. 54, no. 5, pp. 794-797, 2006.

[20] A. M. Tonello, "Performance limits for filtered multitone modulation in fading channels," IEEE Transactions on Wireless Communications, vol. 4, no. 5, pp. 2121-2135, 2005.

[21] A. M. Tonello and F. Pecile, "Analytical results about the robustness of FMT modulation with several prototype pulses in time-frequency selective fading channels," IEEE Transactions on Wireless Communications, vol. 7, no. 5, pp. 1634-1645, 2008 . 
[22] N. Moret and A. Tonello, "Similarities and differences among filtered multitone modulation realizations and orthogonal filter bank design," in Proceeding of the 17th European Signal Processing Conference (EUSIPCO '09), Glasgow, Scotland, August 2009.

[23] V. Ercegl, et al., "IEEE P802.11 Wireless LANs, TGn Channel Models," doc.: IEEE 802.11-03/940r4, May 2004.

[24] A. V. Oppenheim and R. W. Schafer, Digital Signal Processing, Prentice-Hall, Upper Saddle River, NJ, USA, 1989.

[25] N. Mizutani, S. Muramatsu, and H. Kikuchi, "Memory access estimation of filter bank implementation on different DSP architectures," IEICE Transactions on Fundamentals of Electronics, Communications and Computer Sciences, vol. E84A, no. 8, pp. 1951-1959, 2001.

[26] M. Vetterli and J. Kovacevic, Wavelet and Subband Coding, Prentice-Hall, Upper Saddle River, NJ, USA, 1995. 